\title{
FULL INDEFINITE STIELTJES MOMENT PROBLEM AND PADÉ APPROXIMANTS
}

\author{
VOLODYMYR DERKACH AND IVAN KOVALYOV
}

Dedicated to Yu. M. Arlinskii on the occasion of his 70th birthday

\begin{abstract}
Full indefinite Stieltjes moment problem is studied via the step-by-step Schur algorithm. Naturally associated with indefinite Stieltjes moment problem are generalized Stieltjes continued fraction and a system of difference equations, which, in turn, lead to factorization of resolvent matrices of indefinite Stieltjes moment problem. A criterion for such a problem to be indeterminate in terms of continued fraction is found and a complete description of its solutions is given in the indeterminate case. Explicit formulas for diagonal and sub-diagonal Padé approximants for formal power series corresponding to indefinite Stieltjes moment problem and convergence results for Padé approximants are presented.
\end{abstract}

\section{INTRODUCTION}

The classical Stieltjes moment problem consists in the following: given a sequence of real numbers $s_{j}\left(j \in \mathbb{Z}_{+}:=\mathbb{N} \cup\{0\}\right)$ find a positive measure $\sigma$ with a support on $\mathbb{R}_{+}$, such that

$$
\int_{\mathbb{R}_{+}} t^{j} d \sigma(t)=s_{j}, \quad j \in \mathbb{Z}_{+}
$$

It follows easily from (1.1) that the inequalities

$$
S_{n}:=\left(s_{i+j}\right)_{i, j=0}^{n-1} \geq 0 \quad \text { and } \quad S_{n}^{+}:=\left(s_{i+j+1}\right)_{i, j=0}^{n-1} \geq 0, \quad n \in \mathbb{Z}_{+},
$$

are necessary for solvability of the moment problem (1.1). Moreover, the inequalities (1.2) are also sufficient for solvability of the moment problem (1.1), see [1, Appendix, Section A1]. Let

$$
D_{n}:=\operatorname{det} S_{n} \quad \text { and } \quad D_{n}^{+}:=\operatorname{det} S_{n}^{+}, \quad n \in \mathbb{Z}_{+} .
$$

For every solution $\sigma$ of the Stieltjes moment problem its Stieltjes transform

$$
f(z)=\int_{\mathbb{R}_{+}} \frac{d \sigma(t)}{t-z}, \quad z \in \mathbb{C} \backslash \mathbb{R}_{+}
$$

belongs to the class $\mathbf{N}$ of functions holomorphic on $\mathbb{C} \backslash \mathbb{R}$ with nonnegative imaginary part in $\mathbb{C}_{+}$and such that $f(\bar{z})=\overline{f(z)}$ for $z \in \mathbb{C}_{+}$. Moreover, $f$ belongs to the Stieltjes class $\mathbf{S}$ of functions $f \in \mathbf{N}$, which admit holomorphic and nonnegative continuation to $\mathbb{R}_{-}$. By M. G. Krein's criterion [27]

$$
f \in \mathbf{S} \Longleftrightarrow f \in \mathbf{N} \text { and } z f \in \mathbf{N} .
$$

2020 Mathematics Subject Classification. Primary 30E05; Secondary 15B57, 30B70, 46C20.

Key words and phrases. Indefinite Stieltjes moment problem, generalized Stieltjes function, generalized Stieltjes polynomials, Schur algorithm, resolvent matrix.

The present research was supported by Ministry of Education and Science of Ukraine (projects \# 0118U003138, 0118U002060), and a grant of the Volkswagen Foundation. V. D. also gratefully acknowledges financial support by the German Research Foundation (DFG, grant TR 903/22-1). 
Notice, that by the Hamburger-Nevanlinna theorem [1, Theorem 3.2.1] the Stieltjes moment problem can be reformulated as the following interpolation problem at $\infty$ for the Stieltjes transform $f(z)=\int_{\mathbb{R}_{+}} \frac{d \sigma(t)}{t-z}$ of the measure $\sigma$

$$
f(z)=-\frac{s_{0}}{z}-\frac{s_{1}}{z^{2}}-\cdots-\frac{s_{2 n}}{z^{2 n+1}}+o\left(\frac{1}{z^{2 n+1}}\right), \quad z \widehat{\rightarrow} \infty .
$$

The notation $z \widehat{\rightarrow} \infty$ means that $z \rightarrow \infty$ nontangentially, that is inside the sector $\varepsilon<$ $\arg z<\pi-\varepsilon$ for some $\varepsilon>0$.

In the pioneering paper [40] by T. Stieltjes it was shown that if $D_{n}, D_{n}^{+}$are positive for all $n \in \mathbb{Z}_{+}$and

$$
l_{n}:=\frac{D_{n}^{2}}{D_{n}^{+} D_{n-1}^{+}}, \quad m_{n}:=\frac{\left(D_{n-1}^{+}\right)^{2}}{D_{n} D_{n-1}}, \quad n \in \mathbb{N},
$$

then the Laurent series $-\sum_{j=0}^{\infty} \frac{s_{j}}{z^{j+1}}$ can be uniquely developed in the continued fraction

$$
\frac{1}{-z m_{1}+\frac{1}{l_{1}+\ldots \frac{1}{-z m_{n}+\frac{1}{l_{n}+\ldots}}}} \text {. }
$$

The moment problem (1.1) is called determinate, if it has unique solution, and indeterminate otherwise. A solution $\sigma$ of (1.1) is called canonical, if the set of polynomials is dense in $L^{2}(\sigma)$. As was shown in [40] the moment problem (1.1) is indeterminate, if and only if

$$
M:=\sum_{i=1}^{\infty} m_{i}<\infty \quad \text { and } \quad L:=\sum_{i=1}^{\infty} l_{i}<\infty .
$$

As was shown in $\left[27\right.$, Appendix, $\left.13.2^{\circ}\right]$, in the indeterminate case there exists an entire matrix valued function $W(z)=\left(w_{i, j}(z)\right)_{i, j=1}^{2}$ such that the set of all solutions of the problem (1.1) can be parametrised by the formula

$$
\int_{\mathbb{R}_{+}} \frac{d \sigma(t)}{t-z}=T_{W(z)}[\tau(z)]:=\frac{w_{11}(z) \tau(z)+w_{12}(z)}{w_{21}(z) \tau(z)+w_{22}(z)},
$$

where $\tau$ ranges over the Stieltjes class $\mathbf{S}$. A subset of its canonical solutions was described already in [40].

Now let us remind the indefinite versions of the classes $\mathbf{N}$ and $\mathbf{S}$, see [30].

Definition 1.1. ([30]) A function $f$ meromorphic on $\mathbb{C} \backslash \mathbb{R}$ with the set of holomorphy $\mathfrak{h}_{f}$ is said to be in the generalized Nevanlinna class $\mathbf{N}_{\kappa}(\kappa \in \mathbb{N})$, if for every set $z_{j} \in \mathbb{C}_{+} \cap \mathfrak{h}_{f}$ $\left(z_{i} \neq \bar{z}_{j}, i, j=1, \ldots, n\right)$ the form

$$
\sum_{i, j=1}^{n} \frac{f\left(z_{i}\right)-\overline{f\left(z_{j}\right)}}{z_{i}-\bar{z}_{j}} \xi_{i} \bar{\xi}_{j}
$$

has at most $\kappa$ and for some choice of $z_{j}(j=1, \ldots, n)$ exactly $\kappa$ negative squares. A functions $f \in \mathbf{N}_{\kappa}$ is said to belong to the generalized Stieltjes class $\mathbf{N}_{\kappa}^{+}$, if $z f \in \mathbf{N}$.

Similarly, in $[9,10]$ the class $\mathbf{N}_{\kappa}^{k}(\kappa, k \in \mathbb{N})$ was introduced as the set of functions $f \in \mathbf{N}_{\kappa}$, such that $z f(z)$ belongs to the class $\mathbf{N}_{k}$, see also $[18,20]$, where the class $\mathbf{N}_{0}^{k}$ was studied.

In the present paper we consider the following problems.

Full indefinite moment problem $M P_{\kappa}(\mathbf{s})$. Given $\kappa \in \mathbb{Z}_{+}$, and an infinite sequence $\mathbf{s}=$ $\left\{s_{j}\right\}_{j=0}^{\infty}$ of real numbers, describe the set $\mathcal{M}_{\kappa}(\mathbf{s})$ of functions $f \in \mathbf{N}_{\kappa}$, which satisfy (1.10) for all $\ell \in \mathbb{N}$.

Full indefinite moment problem $M P_{\kappa}^{k}(\mathbf{s})$. Given $\kappa, k \in \mathbb{Z}_{+}$, and an infinite sequence $\mathbf{s}=\left\{s_{j}\right\}_{j=0}^{\infty}$ of real numbers, describe the set $\mathcal{M}_{\kappa}^{k}(\mathbf{s}):=\mathcal{M}_{\kappa}(\mathbf{s}) \cap \mathbf{N}_{\kappa}^{k}$. 
Indefinite moment problems $M P_{\kappa}(\mathbf{s})$ and $M P_{\kappa}^{+}(\mathbf{s})$ were studied in [31], [32] by the methods of extension theory of Pontryagin space symmetric operators developed in [30], [31]. In particular, it was shown in [31] that the moment problem $M P_{\kappa}^{+}(\mathbf{s})$ is solvable if and only if the number $\nu_{-}\left(S_{n}\right)$ of negative eigenvalues of $S_{n}$ does not exceed $\kappa$ and $S_{n}^{+}>0$ for all $n \in \mathbb{N}$. Further applications of the operator approach to the moment problem $M P_{\kappa}^{k}(\mathbf{s})$ were given in [10]. A reproducing kernel approach to the moment problems $M P_{\kappa}(\mathbf{s})$ was presented in [21]. A step-by-step algorithm of solving the moment problems $M P_{\kappa}(\mathbf{s})$ was elaborated in [6], [7] and [2]. Applications of the Schur algorithm to degenerate moment problem in the class $\mathbf{N}_{\kappa}$ were given in [14].

The step-by-step algorithm reduces the moment problems $M P_{\kappa}(\mathbf{s})$ and $M P_{\kappa}^{k}(\mathbf{s})$ to the following truncated moment problems:

Truncated indefinite moment problem $M P_{\kappa}(\mathbf{s}, \ell)$. Given $\ell, \kappa \in \mathbb{Z}_{+}$, and a finite sequence $\mathbf{s}=\left\{s_{j}\right\}_{j=0}^{\ell}$ of real numbers, describe the set $\mathcal{M}_{\kappa}(\mathbf{s}, \ell)$ of functions $f \in \mathbf{N}_{\kappa}$, which satisfy the asymptotic expansion

$$
f(z)=-\frac{s_{0}}{z}-\cdots-\frac{s_{\ell}}{z^{\ell+1}}+o\left(\frac{1}{z^{\ell+1}}\right), \quad z=i y, \quad y \uparrow \infty .
$$

Truncated indefinite moment problem $M P_{\kappa}^{k}(\mathbf{s}, \ell)$. Given $\ell, \kappa, k \in \mathbb{Z}_{+}$, and a sequence $\mathbf{s}=\left\{s_{j}\right\}_{j=0}^{\ell}$ of real numbers, describe the set $\mathcal{M}_{\kappa}^{k}(\mathbf{s}, \ell)$ of functions $f \in \mathbf{N}_{\kappa}^{k}$, which satisfy (1.10). A truncated moment problem is called even or odd regarding to the oddness of the number $\ell+1$ of given moments.

Let $\mathcal{H}$ be the set of all infinite real sequences $\mathbf{s}=\left\{s_{j}\right\}_{j=0}^{\infty}$ and let $\mathcal{H}_{\kappa}$ be the set of sequences $\mathbf{s} \in \mathcal{H}$, such that

$$
\nu_{-}\left(S_{n}\right)=\kappa \text { for all } n \text { big enough. }
$$

Denote by $\mathcal{H}_{\kappa}^{k}$ the set of real sequences $\mathbf{s} \in \mathcal{H}_{\kappa}$, such that $\left\{s_{j+1}\right\}_{j=0}^{\infty} \in \mathcal{H}_{k}$, i.e.

$$
\nu_{-}\left(S_{n}^{+}\right)=k \quad \text { for all } n \text { big enough. }
$$

A number $n_{j} \in \mathbb{N}$ is said to be a normal index of the sequence $\mathbf{s} \in \mathcal{H}$, if $\operatorname{det} S_{n_{j}} \neq 0$. The ordered set of normal indices

$$
n_{1}<n_{2}<\cdots<n_{N}
$$

of the sequence $\mathbf{s}$ is denoted by $\mathcal{N}(\mathbf{s})$.

As was shown in [7] for every $\mathbf{s} \in \mathcal{H}_{\kappa}$ there exists a sequence of real numbers $b_{i} \in$ $\mathbb{R} \backslash\{0\}, i \in \mathbb{N}$ and real monic polynomials

$$
a_{i}(z)=z^{\ell_{i}}+a_{\ell_{i}-1}^{(i)} z^{\ell_{i}-1}+\cdots+a_{1}^{(i)} z+a_{0}^{(i)}
$$

of degree $\ell_{i}=n_{i+1}-n_{i}, i \in \mathbb{N}$, such that the convergents of the continued fraction

$$
\frac{-b_{0}}{a_{0}(z)-\frac{b_{1}}{a_{1}(z)-\cdots-\frac{b_{n}}{a_{n}(z)-\ldots}}}
$$

for sufficiently large $n$ have the asymptotic expansion (1.10) for every $\ell \in \mathbb{N}$. This fact was known already to L. Kronecker [33] and then it was reinvented in [6]. The pairs $\left(a_{i}, b_{i}\right)$ are called atoms, see [24] and the continued fraction (1.14) is called the $P$-fraction, [36].

Consider the three-term recurrence relation

$$
b_{j} y_{j-1}(z)-a_{j}(z) y_{j}(z)+y_{j+1}(z)=0
$$

associated with the sequence of atoms $\left\{a_{i}, b_{i}\right\}, i \in \mathbb{N}$, and define polynomials $P_{j}(z)$ and $Q_{j}(z)$ as solutions of the system (1.15) subject to the initial conditions

$$
P_{-1}(z) \equiv 0, \quad P_{0}(z) \equiv 1, \quad Q_{-1}(z) \equiv-1, \quad Q_{0}(z) \equiv 0 .
$$


The polynomials $P_{j}$ and $Q_{j}$ are called Lanzcos polynomials of the first and second kind. Moreover, the $n$-th convergent of the continued fraction (1.14) takes the form (see [24, Section 8.3.7]).

$$
f^{[n]}(z)=-\frac{Q_{n}(z)}{P_{n}(z)}, \quad n \in \mathbb{N} .
$$

As was shown in [7] the set $\mathcal{M}_{\kappa}\left(\mathbf{s}, 2 n_{j}-2\right)$ can be described in terms of the Lanzcos polynomials of the first and second kind.

A sequence $\mathbf{s} \in \mathcal{H}_{\kappa}^{k}$ is called regular (see [15]), and is designated as $\mathbf{s} \in \mathcal{H}_{\kappa}^{k, r e g}$, if

$$
D_{n_{j}}^{+}=\operatorname{det} S_{n_{j}}^{+} \neq 0 \text { for all } j \in \mathbb{N} .
$$

In [16] it was shown that an even indefinite Stieltjes moment problems $M P_{\kappa}^{k}\left(\mathbf{s}, 2 n_{N}-1\right)$ for regular sequence $\mathbf{s}$ is solvable if and only if

$$
\kappa_{N}:=n_{-}\left(S_{n_{N}}\right) \leq \kappa \text { and } k_{N}^{+}:=n_{-}\left(S_{n_{N}}^{+}\right) \leq k .
$$

For this problem one step of the Schur algorithm was split in [16] into two substeps and this leads to the expansion of $f \in \mathcal{M}_{\kappa}^{k}\left(\mathbf{s}, 2 n_{N}-2\right)$ into a generalized Stieltjes continued fraction

$$
f(z)=\frac{1}{-z m_{1}(z)+\frac{1}{l_{1}+\cdots+\frac{1}{-z m_{N}(z)+\frac{1}{l_{N}+f_{N}(z)}}}},
$$

where $m_{j}$ are polynomials, $l_{j} \in \mathbb{R} \backslash\{0\}$ and $f_{N}$ is a function from the generalized Stieltjes class $\mathbf{N}_{\kappa-\kappa_{N}}^{k-k_{N}}$, such that $f_{N}(z)=o(1)$ as $z \widehat{\rightarrow} \infty$. If $f \in \mathcal{M}_{\kappa}^{k}(\mathbf{s})$ then $f_{N}$ has an induced asymptotic expansion

$$
f_{N}(z)=-\frac{s_{0}^{(N)}}{z}-\frac{s_{1}^{(N)}}{z^{2}}-\cdots-\frac{s_{2 n}^{(N)}}{z^{2 n+1}}+o\left(\frac{1}{z^{2 n+1}}\right), \quad z \widehat{\rightarrow} \infty
$$

i.e. $f_{N}$ is a solution of an induced moment problem $M P_{\kappa-\kappa_{N}}^{k-k_{N}}\left(\mathbf{s}^{(N)}\right)$ generated by the sequence $\mathbf{s}^{(N)}=\left(s_{i}^{(N)}\right)_{i=0}^{\infty}$. Then $f_{N} \in \mathcal{M}_{\kappa}^{k}\left(\mathbf{s}^{(N)}\right)$.

Generalized Stieltjes continued fractions were studied in [15]. Associated to the continued fraction (1.19) there is a system of difference equations (see [42, Section 1])

$$
\left\{\begin{array}{l}
y_{2 j-1}-y_{2 j-3}=-z m_{j}(z) y_{2 j-2}, \quad j \in \mathbb{N} . \\
y_{2 j}-y_{2 j-2}=l_{j} y_{2 j-1},
\end{array}\right.
$$

Define the generalized Stieltjes polynomials $P_{j}^{+}$and $Q_{j}^{+}$of the first and second kind as solutions of the system (1.21) subject to the initial conditions

$$
P_{-1}^{+}(z) \equiv 0, \quad P_{0}^{+}(z) \equiv 1, \quad Q_{-1}^{+}(z) \equiv 1, \quad Q_{0}^{+}(z) \equiv 0 .
$$

The formula (1.19) for the set of solutions of the truncated Stieltjes moment problem $M P_{\kappa}^{k}\left(\mathbf{s}, 2 n_{N}-1\right)$ can be rewritten in terms of the generalized Stieltjes polynomials $P_{j}^{+}$ and $Q_{j}^{+}, j=2 N-1,2 N$.

Theorem 1.2. ([17]) Let a sequence $\mathbf{s}=\left\{s_{i}\right\}_{i=0}^{\infty} \in \mathcal{H}_{\kappa}^{k}$ be regular, $\mathcal{N}(\mathbf{s})=\left\{n_{j}\right\}_{j=1}^{\infty}$, and let $P_{j}^{+}$and $Q_{j}^{+}$be generalized Stieltjes polynomials of the first and second kind. Then

(i) A nondegenerate even moment problem $M P_{\kappa}^{k}\left(s, 2 n_{N}-1\right)$ is solvable, if and only if (1.18) holds.

(ii) $f \in \mathcal{M}_{\kappa}^{k}\left(s, 2 n_{N}-1\right)$ if and only if $f$ admits the representation

$$
f(z)=\frac{Q_{2 N-1}^{+}(z) f_{N}(z)+Q_{2 N}^{+}(z)}{P_{2 N-1}^{+}(z) f_{N}(z)+P_{2 N}^{+}(z)}
$$

where $f_{N}(z)$ satisfies the conditions

$$
f_{N} \in \mathbf{N}_{\kappa-\kappa_{N}}^{k-k_{N}^{+}} \quad \text { and } \quad f_{N}(z)=o(1), \quad z \widehat{\rightarrow} \infty .
$$


In what follows for every $2 \times 2$ matrix $W=\left(w_{i j}\right)_{i, j=1}^{2}$ we associate the linear-fractional transformation

$$
T_{W}[\tau]:=\frac{w_{11} \tau+w_{12}}{w_{21} \tau+w_{22}}
$$

Denote by $W_{2 N}(z)$ the coefficient matrix of the linear fractional transform (1.23):

$$
W_{2 N}(z)=\left(\begin{array}{cc}
Q_{2 N-1}^{+}(z) & Q_{2 N}^{+}(z) \\
P_{2 N-1}^{+}(z) & P_{2 N}^{+}(z)
\end{array}\right) .
$$

Then the formula (1.23) can be rewritten as

$$
f(z)=T_{W_{2 N}(z)}\left[f_{N}(z)\right]
$$

The structure of the continued fraction (1.19) leads to the following factorization of the matrix valued function $W_{2 N}(z)$ :

$$
W_{2 N}(z)=M_{1}(z) L_{1} \ldots M_{N}(z) L_{N}
$$

where the matrices $M_{j}(z)$ and $L_{j}$ are defined by

$$
M_{j}(z)=\left(\begin{array}{cc}
1 & 0 \\
-z m_{j}(z) & 1
\end{array}\right) \quad \text { and } \quad L_{j}=\left(\begin{array}{cc}
1 & l_{j} \\
0 & 1
\end{array}\right), \quad j \in \mathbb{N} .
$$

Continued fractions of the form (1.19) with positive and negative masses $m_{j}$ were studied by Beals, Sattinger and Szmigielski [4] in connection with the theory of multipeakon solutions of the Camassa-Holm equation. In [22] Eckhardt and Kostenko showed that inverse spectral problem for multi-peakon solutions of the Camassa-Holm equation is solvable in the class of continued fractions of the form (1.19) with polynomials $m_{j}(z)=$ $d_{j} z+m_{j}$ of formal degree 1. In [23] the spectral theory of continued fractions (1.19) was treated within a classical Hamburger moment problem associated with this sequence.

The full indefinite Stieltjes moment problem $M P_{\kappa}^{k}(\mathbf{s})$ for regular sequences $\mathbf{s} \in \mathcal{H}_{\kappa}^{k, r e g}$ was considered in [17] via the operator approach. The moment problem $M P_{\kappa}^{k}(\mathbf{s})$ was treated in [17] as a problem of extension theory for a symmetric operator generated by this generalized Jacobi matrix. In that paper we used quite advanced tools: the theory of boundary triples developed in [25, 18, 19], and the M. G. Krĕn theory of resolvent matrices extended in $[9,12]$ to the case of indefinite inner spaces.

In the present paper we are going to use elementary tools in order to make the presentation available for a wider audience. The main idea is to use the factorization formula for the coefficients matrix $W_{2 j}(z)$

$$
W_{2 j}(z)=W_{2 N}(z) W_{2(j-N)}^{(N)}(z), \quad j>N, \quad j, N \in \mathbb{N},
$$

which allows to reduce the indefinite Stieltjes moment problem $M P_{\kappa}^{k}(\mathbf{s}, 2 j)$ to some classical Stieltjes moment problem $M P_{0}^{0}\left(\mathbf{s}^{(N)}, 2(j-N)\right)$ with the resolvent matrix $W_{2(j-N)}^{(N)}(z)$. Then all the known results for the classical Stieltjes moment problem can be translated to the indefinite Stieltjes moment problem. In particular, in Theorem 4.2 it is shown that the problem $M P_{\kappa}^{k}(\mathbf{s})$ is indeterminate if and only if

$$
M:=\sum_{j=1}^{\infty} m_{j}(0)<\infty \text { and } L:=\sum_{j=1}^{\infty} l_{j}<\infty .
$$

In the classical case, polynomials $m_{j}(z)$ are constants and the criterion (1.30) coincides with the well known Stieltjes criterion, see [1, Theorem 0.4].

If (1.30) is in force, then the matrix valued functions $W_{2 j}(z)$ converge to an entire matrix valued function $W_{\infty}^{+}(z)$ of order $1 / 2$ and the linear fractional transformation (1.27) generated by the matrix valued function $W_{\infty}(z)$ provides a description of the set $\mathcal{M}_{\kappa}^{k}(\mathbf{s})$.

In Section 5 Padé approximants for formal power series corresponding to an indefinite Stieltjes moment problem are calculated. As was shown in [8] the diagonal Padé approximants for formal power series corresponding to an indefinite Hamburger moment problem are represented as a ratio of the Lanzcos polynomials of the 2-nd and the 1-st 
kind, [8]. In Theorem 5.4 we show that the sub-diagonal Padé approximants of the corresponding formal power series is a ratio of the generalized Stieltjes polynomials of the 2-nd and the 1-st kind. In Theorem 5.6 convergence of Padé approximants is derived from the classical results using the formula (1.29).

In Section 6 the results are illustrated by an example of indefinite moment problem associated with Laguerre polynomials $L_{n}(z, \alpha)$ in the non-classical case $\alpha<-1$.

\section{Preliminaries}

2.1. Generalized Nevanlinna functions. A function $f \in \mathbf{N}_{\kappa}$ is said to belong to the class $\mathbf{N}_{\kappa,-\ell}\left(\kappa, \ell \in \mathbb{Z}_{+}:=\mathbb{N} \cup\{0\}\right)$ if $f$ admits the asymptotic expansion (1.10) for some real numbers $s_{0}, \ldots, s_{\ell}$. Let us also set

$$
\mathbf{N}_{\kappa,-\infty}:=\bigcap_{n \geq 0} \mathbf{N}_{\kappa,-2 n}
$$

Every real polynomial $P(z)=p_{\nu} z^{\nu}+\cdots+p_{1} z+p_{0}$ of degree $\nu$ belongs to a class $\mathbf{N}_{\kappa_{-}(P)}$, where the index $\kappa_{-}(P)$ can be evaluated by (see [30, Lemma 3.5])

$$
\kappa_{-}(P)= \begin{cases}{\left[\frac{\nu+1}{2}\right],} & \text { if } p_{\nu}<0 ; \text { and } \nu \text { is odd }, \\ {\left[\frac{\nu}{2}\right],} & \text { otherwise } .\end{cases}
$$

Recall, that a function $f \in \mathbf{N}_{\kappa}$ is said to be from the generalized Stieltjes class $\mathbf{N}_{\kappa}^{ \pm k}$, if $z^{ \pm 1} f(z)$ belongs to $\mathbf{N}_{k}\left(\kappa, k \in \mathbb{Z}_{+}\right)$. Let us collect some properties of generalized Nevanlinna functions, see [30], [9].

Proposition 2.1. ([30]) Let $\kappa, \kappa_{1}, k \in \mathbb{Z}_{+}$. Then the following statements hold:

(i) $f \in \mathbf{N}_{\kappa} \Longleftrightarrow-\frac{1}{f} \in \mathbf{N}_{\kappa}$;

(ii) $f \in \mathbf{N}_{\kappa}^{k} \Longleftrightarrow-\frac{1}{f} \in \mathbf{N}_{\kappa}^{-k}$;

(iii) $f \in \mathbf{N}_{\kappa}^{k} \Longleftrightarrow z f(z) \in \mathbf{N}_{k}^{-\kappa}$;

(iv) if $f \in \mathbf{N}_{\kappa}, f_{1} \in \mathbf{N}_{\kappa_{1}}$ then $f+f_{1} \in \mathbf{N}_{\kappa^{\prime}}$, where $\kappa^{\prime} \leq \kappa+\kappa_{1}$. If, in addition, $f(i y)=o(y)$ as $y \rightarrow \infty$ and $f_{1}$ is a polynomial, then $f+f_{1} \in \mathbf{N}_{\kappa+\kappa_{1}}$;

(v) if a function $f \in \mathbf{N}_{\kappa}$ has an asymptotic expansion (1.5) for every $n \in \mathbb{N}$, then there exists $\kappa^{\prime} \leq \kappa$, such that $\left\{s_{j}\right\}_{j=0}^{\infty} \in \mathcal{H}_{\kappa^{\prime}}$.

The notions of generalized poles of non-positive type of a function $f \in \mathbf{N}_{\kappa}$ were introduced in [32]. The following definitions are based on [34]. A point $\alpha \in \mathbb{R}$ is called a generalized pole of non-positive type (GPNT) of the function $f \in \mathbf{N}_{\kappa}$ with multiplicity $\kappa_{\alpha}(f)$ if

$$
-\infty<\lim _{z \widehat{\rightarrow} \alpha}(z-\alpha)^{2 \kappa_{\alpha}+1} f(z) \leq 0, \quad 0<\lim _{z \rightarrow \alpha}(z-\alpha)^{2 \kappa_{\alpha}-1} f(z) \leq \infty .
$$

Similarly, the point $\infty$ is called a generalized pole of $f$ of nonpositive type (GPNT) with multiplicity $\kappa_{\infty}(f)$ if

$$
0 \leq \lim _{z \widehat{\rightarrow} \infty} \frac{f(z)}{z^{2 \kappa_{\infty}+1}}<\infty, \quad-\infty \leq \lim _{z \rightrightarrows \infty} \frac{f(z)}{z^{2 \kappa_{\infty}-1}}<0 .
$$

The following fundamental result was proved in [32, Theorem 3.5].

Proposition 2.2. Let $f \in \mathbf{N}_{\kappa}$. Then the total multiplicity of the poles of $f$ in $\mathbb{C}_{+}$and the generalized poles of negative type of $f$ in $\mathbb{R} \cup\{\infty\}$ is equal to $\kappa$.

2.2. Regular sequences and step-by-step algorithm. In the present paper we will consider so-called regular sequences $\mathbf{s}$ from $\mathcal{H}_{\kappa}^{k}$ introduced in [15].

Definition 2.3. The sequence $\mathbf{s}$ is called regular (and is denoted as $\mathbf{s} \in \mathcal{H}_{\kappa}^{k, r e g}$ ), if one of the following equivalent conditions holds:

(i) $P_{j}(0) \neq 0$ for every $j \in \mathbb{N}$;

(ii) $D_{n_{j}}^{+}:=\operatorname{det} S_{n_{j}}^{+} \neq 0$ for every $j \in \mathbb{N}$;

(iii) $D_{n_{j}-1}^{+} \neq 0$ for every $j \in \mathbb{N}$. 
The following step-by-step algorithm of solving the indefinite Stieltjes moment problem for regular sequences $\mathbf{s} \in \mathcal{H}_{\kappa}^{k, r e g}$ was developed in [17]. For an indefinite Hamburger moment problem such an algorithm was elaborated in $[6,7]$.

Let $f \in \mathcal{M}_{\kappa}^{k}(\mathbf{s})$ and let $n_{1}$ be the first normal index, i.e.

$$
s_{0}=\cdots=s_{n_{1}-2}=0, \quad s_{n_{1}-1} \neq 0 .
$$

By Proposition $2.1(4)-1 / f \in \mathbf{N}_{\kappa}^{-k}$ and

$$
-\frac{1}{f(z)}=P_{1}(z)+o(1) \quad \text { as } \quad z \widehat{\rightarrow} \infty
$$

where $P_{1}(z)$ is the Lanzcos polynomial of degree: $\operatorname{deg} P_{1}=n_{1}$. Since $\mathbf{s}$ is regular, then $-l_{1}^{-1}:=P_{1}(0) \neq 0$ and hence $(2.5)$ can be rewritten as

$$
-\frac{1}{f(z)}=z m_{1}(z)-\frac{1}{g_{1}(z)}=z m_{1}(z)-\frac{1}{l_{1}+f_{1}(z)} .
$$

Let $\kappa_{1}=\kappa_{-}\left(z m_{1}\right), k_{1}=\kappa_{-}\left(m_{1}\right), k_{1}^{+}=k_{1}+\kappa_{-}\left(z l_{1}\right)$. Then by Proposition $2.1(2),(4)$

$$
z m_{1} \in \mathbf{N}_{\kappa_{1}}^{-k_{1}} \Rightarrow-\frac{1}{g_{1}}=-\frac{1}{f}-z m_{1} \in \mathbf{N}_{\kappa-\kappa_{1}}^{-\left(k-k_{1}\right)} \Rightarrow g_{1} \in \mathbf{N}_{\kappa-\kappa_{1}}^{k-k_{1}} \Rightarrow f_{1} \in \mathbf{N}_{\kappa-\kappa_{1}}^{k-k_{1}^{+}} .
$$

The formula (2.6) yields the following representation of $f \in \mathcal{M}_{\kappa}^{k}(\mathbf{s})$ :

$$
f(z)=\frac{1}{-z m_{1}(z)+\frac{1}{l_{1}+f_{1}(z)}},
$$

where $f_{1} \in \mathbf{N}_{\kappa-\kappa_{1}}^{k-k_{1}^{+}}$satisfies the asymptotic expansion

$$
f_{1}(z)=-\frac{s_{0}^{(1)}}{z}-\frac{s_{1}^{(1)}}{z^{2}}-\cdots-\frac{s_{2 n}^{(1)}}{z^{2 n+1}}+o\left(\frac{1}{z^{2 n+1}}\right), \quad z \widehat{\rightarrow} \infty
$$

with some real numbers $s_{i}^{(1)}, i \in \mathbb{Z}_{+}$. Explicit formulas for calculation of the sequence $\mathbf{s}^{(1)}=\left\{s_{i}^{(1)}\right\}_{i=0}^{\infty}$ are presented in [17, Remarks 3.4, 3.6], the polynomial $m_{1}(z)$ and the number $l_{1}$ can be found by (see $\left.[17,(2.16),(3.27)]\right)$ :

$$
\begin{gathered}
m_{1}(z)=\frac{(-1)^{n_{1}+1}}{D_{n_{1}}}\left|\begin{array}{ccccc}
0 & \ldots & 0 & s_{n_{1}-1} & s_{n_{1}} \\
\vdots & & \ldots & \ldots & \vdots \\
s_{n_{1}-1} & \ldots & \ldots & \ldots & s_{2 n_{1}-2} \\
1 & z & \ldots & z^{n_{1}-2} & z^{n_{1}-1}
\end{array}\right|, \\
l_{1}=(-1)^{n_{1}+1} s_{n_{1}-1} \frac{D_{n_{1}}}{D_{n_{1}}^{+}}, \quad D_{\nu}:=\operatorname{det} S_{\nu}, \quad D_{\nu}^{+}:=\operatorname{det} S_{\nu}^{+} .
\end{gathered}
$$

This completes the first step of the Schur algorithm.

Applying this algorithm repeatedly $N$ times one obtains a function $f_{N} \in \mathbf{N}_{\kappa-\kappa_{N}}^{k-k_{N}^{+}}$, with $\kappa_{N}$ and $k_{N}$ given by (1.18), connected with $f$ by the formula (1.19). Moreover, the function $f_{N}$ satisfies the asymptotic expansion

$$
f_{N}(z)=-\frac{s_{0}^{(N)}}{z}-\frac{s_{1}^{(N)}}{z^{2}}-\cdots-\frac{s_{2 n}^{(N)}}{z^{2 n+1}}+o\left(\frac{1}{z^{2 n+1}}\right), \quad z \widehat{\rightarrow} \infty
$$

with some real $s_{i}^{(N)}, i \in \mathbb{Z}_{+}$. 
Lemma 2.4. ([15]) Let $\mathbf{s} \in \mathcal{H}_{\kappa}^{k, r e g}$. Then there exist sequences of polynomials $m_{j}(z)$ and numbers $l_{j}$ such that the $2 j$-th convergent $\frac{u_{2 j}}{v_{2 j}}$ of the continued fraction

$$
\frac{1}{-z m_{1}(z)+\frac{1}{l_{1}+\ldots \frac{1}{-z m_{j}(z)+\frac{1}{l_{j}+\ldots}}}}
$$

coincides with the $j$-th convergent of the P-fraction (1.14) corresponding to the sequence s. Let functions $f$ and $f_{N}(N \in \mathbb{N})$ be connected by (1.19) and let $\mathbf{s}^{(N)}$ is the $N-$ th induced sequence. Then

$$
\begin{gathered}
f \in \mathcal{M}_{\kappa}^{k}(\mathbf{s}, \ell) \Longleftrightarrow f_{N} \in \mathcal{M}_{\kappa-\kappa_{N}}^{k-k_{N}^{+}}\left(\mathbf{s}^{(N)}, \ell-2 n_{N}\right), \\
f \in \mathcal{M}_{\kappa}^{k}(\mathbf{s}) \Longleftrightarrow f_{N} \in \mathcal{M}_{\kappa-\kappa_{N}}^{k-k_{N}^{+}}\left(\mathbf{s}^{(N)}\right),
\end{gathered}
$$

where $\kappa_{N}$ and $k_{N}^{+}$are given by (1.18).

In the case of a regular sequence $\mathbf{s} \in \mathcal{H}_{\kappa}^{k}$ the parameters $l_{j}$ and $m_{j}(z)$ in (2.12) can be calculated recursively by the above Schur algorithm in terms of the sequence $\mathbf{s}$ :

$$
m_{j}(z)=\frac{(-1)^{\nu+1}}{D_{\nu}^{(j-1)}}\left|\begin{array}{ccccc}
0 & \ldots & 0 & s_{\nu-1}^{(j-1)} & s_{\nu}^{(j-1)} \\
\vdots & & \ldots & \ldots & \vdots \\
s_{\nu-1}^{(j-1)} & \ldots & \ldots & \ldots & s_{2 \nu-2}^{(j-1)} \\
1 & z & \ldots & z^{\nu-2} & z^{\nu-1}
\end{array}\right|
$$

where $D_{\nu}^{(j)}:=\operatorname{det} S_{\nu}^{(j)}, \nu=n_{j}-n_{j-1}$ and

$$
l_{j}=(-1)^{\nu+1} \frac{D_{\nu}^{(j-1)}}{\left(D_{\nu}^{(j-1)}\right)^{+}}, \quad j=1, \ldots, N-1 .
$$

The $N$-th induced sequence $\mathbf{s}^{(N)}$ can be found as the sequence of coefficients of the series expansion $-\sum_{i=0}^{\infty} s_{i}^{(N)} z^{-(i+1)}$ corresponding to the continued fraction

$$
\frac{1}{-z m_{N+1}(z)+\frac{1}{l_{N+1}+\frac{1}{-z m_{N+2}(z)+\frac{1}{\ddots}}} .}
$$

2.3. Generalized Stieltjes continued fractions. In [15] the expansion (2.12) of $f \in$ $\mathcal{M}_{\kappa}^{k}(\mathbf{s})$ into a generalized Stieltjes fraction for $\mathbf{s} \in \mathcal{H}_{\kappa}^{k, r e g}$ was derived from the expansion of its unwrapping transform $z f\left(z^{2}\right)$ into the $P$-fraction.

Theorem 2.5. ([15]) Let $\mathbf{s} \in \mathcal{H}_{\kappa}^{k, r e g}$ and let the P-fraction (1.14) and the generalized $S$-fraction (2.12) correspond to the sequence $\mathbf{s}$. Then the parameters $l_{j}$ and $m_{j}(z)$ $\left(j \in \mathbb{Z}_{+}\right)$of the generalized $\mathbf{S}$-fraction (2.12) are connected with the parameters $b_{j}$ and $a_{j}(z)(j \in \mathbb{N})$ of the $P$-fraction (1.14) by the equalities

$$
\begin{gathered}
b_{0}=\frac{1}{d_{1}}, \quad a_{0}(z)=\frac{1}{d_{1}}\left(z m_{1}(z)-\frac{1}{l_{1}}\right), \\
b_{j}=\frac{1}{l_{j}^{2} d_{j} d_{j+1}}, \quad a_{j}(z)=\frac{1}{d_{j+1}}\left(z m_{j+1}(z)-\left(\frac{1}{l_{j}}+\frac{1}{l_{j+1}}\right)\right),
\end{gathered}
$$

where $d_{j}$ is the leading coefficient of $m_{j}(z)(j=1, \ldots, N-1)$. 
In the case when $m_{j}(z) \equiv m_{j}$ are constants the generalized $S$-fraction reduces to the classical $S$-fraction (1.7) and the formulas (2.18) and (2.19) coincide with the well known classical formulas from [40], see also [1, Appendix, (3), (4)].

Conversely, $m_{j}$ and $l_{j}$ can be represented in terms of the $P$-fractions, see [15, Corollary 4.1$]$.

Corollary 2.6. Let $\mathbf{s} \in \mathcal{H}_{\kappa}^{k, r e g}$, let $\mathbf{s}$ be associated with the $\mathbf{S}$-fraction (2.12), let $d_{i}$ be the leading coefficient of the polynomial $m_{i}(z)$ and let

$$
\widetilde{b}_{i}=b_{0} b_{1} \ldots b_{i}, \quad i \in \mathbb{Z}_{+} .
$$

Then

$$
d_{i}=\frac{P_{i-1}^{2}(0)}{\widetilde{b}_{i-1}}, \quad l_{i}=-\frac{\widetilde{b}_{i-1}}{P_{i-1}(0) P_{i}(0)}, \quad m_{i}(z)=d_{i} \frac{a_{i-1}(z)-a_{i-1}(0)}{z}, \quad i \in \mathbb{N}
$$

Remark 2.7. If $\mathcal{N}(\mathbf{s})=\mathbb{N}$, then $\operatorname{deg}\left(m_{i}\right)=0$ for all $i \in \mathbb{N}$, and $d_{i}=m_{i}, l_{i}$ can be found by (2.21). Alternatively, $m_{i}, l_{i}$ can be found by (1.6).

If $D_{i}^{+}>0$ for all $i \in \mathbb{N}$, then $\operatorname{deg}\left(m_{i}\right) \leq 1$ for all $i \in \mathbb{N}$, and $m_{i}(z)=d_{i} z+m_{i}, l_{i}$ can be found by (2.21). The parameters $d_{i}, m_{i}$ and $l_{i}$ can be also expressed in terms of $D_{i}$ and $D_{i}^{+}$, see [32, Section 5.3], [15, Remark 4.1].

\section{TRUNCATED INDEFINITE MOMENT PROBLEMS}

3.1. A system of difference equations and generalized Stieltjes polynomials. Let us consider a system of difference equations associated with the continued fraction $(2.12)$

$$
\left\{\begin{array}{l}
y_{2 j-1}-y_{2 j-3}=-z m_{j}(z) y_{2 j-2}, \quad j \in \mathbb{N} . \\
y_{2 j}-y_{2 j-2}=l_{j} y_{2 j-1},
\end{array}\right.
$$

If the $j$-th convergent of this continued fraction is denoted by $\frac{u_{j}}{v_{j}}$, then $u_{j}, v_{j}$ can be found as solutions of the system (3.1) (see [42, Section 1]) subject to the following initial conditions

$$
u_{-1} \equiv 1, \quad u_{0} \equiv 0 ; \quad v_{-1} \equiv 0, \quad v_{0} \equiv 1 .
$$

The first two convergents of the continued fraction (2.12) take the form

$$
\frac{u_{1}}{v_{1}}=\frac{1}{-z m_{1}(z)}=T_{M_{1}}[\infty], \quad \frac{u_{2}}{v_{2}}=\frac{l_{1}}{-z l_{1} m_{1}(z)+1}=T_{M_{1} L_{1}}[0] .
$$

Definition 3.1. ([16]) Let $\mathbf{s} \in \mathcal{H}_{\kappa}^{k, r e g}$. Define polynomials $P_{i}^{+}(z), Q_{i}^{+}(z)$ by

$$
\begin{aligned}
& P_{-1}^{+}(z) \equiv 0, \quad P_{0}^{+}(z) \equiv 1, \quad Q_{-1}^{+}(z) \equiv 1, \quad Q_{0}^{+}(z) \equiv 0 \\
& P_{2 i-1}^{+}(z)=-\frac{1}{\widetilde{b}_{i-1}}\left|\begin{array}{ll}
P_{i}(z) & P_{i-1}(z) \\
P_{i}(0) & P_{i-1}(0)
\end{array}\right| \quad \text { and } \quad P_{2 i}^{+}(z)=\frac{P_{i}(z)}{P_{i}(0)}, \\
& Q_{2 i-1}^{+}(z)=\frac{1}{\widetilde{b}_{i-1}}\left|\begin{array}{ll}
Q_{i}(z) & Q_{i-1}(z) \\
P_{i}(0) & P_{i-1}(0)
\end{array}\right| \quad \text { and } \quad Q_{2 i}^{+}(z)=-\frac{Q_{i}(z)}{P_{i}(0)} .
\end{aligned}
$$

The polynomials $P_{i}^{+}(z), Q_{i}^{+}(z)$ are called the generalized Stieltjes polynomials corresponding to the sequence $\mathbf{s}$.

As was noticed in [16] the generalized Stieltjes polynomials coincide with the solutions $u_{i}$ and $v_{i}$ of the system (3.1). 
Proposition 3.2. Let $s \in \mathcal{H}_{\kappa}^{k, r e g}$ and let $P_{j}^{+}(z)$ and $Q_{j}^{+}(z)$ be the generalized Stieltjes polynomials defined by (3.3). Then the solutions $\left\{u_{j}\right\}_{j=0}^{N}$ and $\left\{v_{j}\right\}_{j=0}^{N}$ of the system (3.1), (3.2) take the form

$$
u_{j}=Q_{j}^{+}(z), \quad v_{j}=P_{j}^{+}(z), \quad j=-1,0, \ldots, N .
$$

Remark 3.3. The Stieltjes polynomials satisfy the following properties

$$
P_{2 i-1}^{+}(0)=0, \quad P_{2 i-2}^{+}(0)=1 \quad \text { and } \quad Q_{2 i-1}^{+}(0)=1 .
$$

Obviously, by Definition 3.1

$$
P_{2 i-1}^{+}(0)=-\frac{1}{\widetilde{b}_{i-1}}\left|\begin{array}{ll}
P_{i}(0) & P_{i-1}(0) \\
P_{i}(0) & P_{i-1}(0)
\end{array}\right|=0 \quad \text { and } \quad P_{2 i-2}^{+}(0)=\frac{P_{i}(0)}{P_{i}(0)}=1 .
$$

By (3.7)

$$
Q_{2 i-1}^{+}(0)=\frac{1}{\widetilde{b}_{i-1}}\left|\begin{array}{ll}
Q_{i}(0) & Q_{i-1}(0) \\
P_{i}(0) & P_{i-1}(0)
\end{array}\right|=\frac{Q_{i}(0) P_{i-1}(0)-Q_{i-1}(0) P_{i}(0)}{\widetilde{b}_{i-1}}=1 .
$$

Here we used the generalized Liouville-Ostrogradsky formula (see [17, (2.9)])

$$
Q_{i}(z) P_{i-1}(z)-Q_{i-1}(z) P_{i}(z)=\widetilde{b}_{i-1}, \quad i \in \mathbb{N} .
$$

Lemma 3.4. Let $P_{i}^{+}$and $Q_{i}^{+}$be the Stieltjes polynomials defined by (3.3). Then

$$
P_{2 i}^{+}(z) Q_{2 i-1}^{+}(z)-Q_{2 i}^{+}(z) P_{2 i-1}^{+}(z)=1
$$

Proof. By Definition 3.1 and (3.7) we obtain

$$
\begin{aligned}
P_{2 i}^{+}(z) Q_{2 i-1}^{+}(z)-Q_{2 i}^{+}(z) P_{2 i-1}^{+}(z)= & \frac{P_{i}(z)}{\widetilde{b}_{i-1} P_{i}(0)}\left(Q_{i}(z) P_{i-1}(0)-P_{i}(0) Q_{i-1}(z)\right)- \\
& -\frac{Q_{i}(z)}{\widetilde{b}_{i-1} P_{i}(0)}\left(P_{i}(z) P_{i-1}(0)-P_{i}(0) P_{i-1}(z)\right) \\
= & \frac{Q_{i}(z) P_{i-1}(z)-P_{i}(z) Q_{i-1}(z)}{\widetilde{b}_{i-1}}=1 .
\end{aligned}
$$

This completes the proof.

Lemma 3.5. Let $s \in \mathcal{H}_{\kappa}^{k, \text { reg }}$ and let $P_{i}(z)$ and $Q_{i}(z)\left(i \in \mathbb{Z}_{+}\right)$be Lanczos polynomials of the first and second kind and let $l_{j}$ and $m_{j}(z)(j \in \mathbb{N})$ be parameters of the generalized $\mathbf{S}$-fraction (2.12). Then

(i) The constants $l_{i}$ can be calculated by

$$
l_{i}=-\frac{Q_{i}(0)}{P_{i}(0)}+\frac{Q_{i-1}(0)}{P_{i-1}(0)}, \quad i \in \mathbb{N}
$$

(ii) For every $N \in \mathbb{N}$ the following formulas hold

$$
\begin{gathered}
\sum_{i=1}^{N} l_{i}=-\frac{Q_{N}(0)}{P_{N}(0)}, \quad \sum_{i=1}^{N} d_{i}=\sum_{i=0}^{N-1}\left|P_{i}^{2}(0)\right| \widetilde{b}_{i}^{-1}, \\
\sum_{i=1}^{N} m_{i}(0)=-P_{2 N-1}^{+{ }^{\prime}}(0) .
\end{gathered}
$$

Proof. 1) Let $Q_{i}^{+}(z)$ be Stieltjes polynomials defined by (3.3). Substituting in (3.1) $y_{j}=Q_{j}^{+}$and $z=0$, we obtain

$$
Q_{2 i}^{+}(0)-Q_{2 i-2}^{+}(0)=l_{i} Q_{2 i-1}^{+}(0)
$$


By Definition 3.1 and by the generalized Liouville-Ostrogradsky formula (3.7)

$$
Q_{2 i-1}^{+}(0)=\frac{1}{\widetilde{b}_{i-1}}\left(Q_{i}(0) P_{i-1}(0)-Q_{i-1}(0) P_{i}(0)=1\right.
$$

This implies (3.9).

2) Summing the equalities (3.9) for $i=1, \ldots, N$ one obtains the first equality in (3.10). The second equality in (3.10) is implied by the relation (see [15, Corollary 4.1])

$$
d_{i+1}=\left|P_{i}^{2}(0)\right| \widetilde{b}_{i}^{-1}
$$

3) Differentiating the first equality in (3.1) one obtains

$$
P_{2 i-1}^{+^{\prime}}(z)=-m_{i}(z) P_{2 i-2}^{+}(z)-z\left(m_{i}(z) P_{2 i-2}^{+}(z)\right)^{\prime}+P_{2 i-3}^{+^{\prime}}(z) .
$$

Substituting $z=0$ and using the equality $P_{2 i-2}^{+}(0)=1$, one obtains

$$
m_{i}(0)=P_{2 i-3}^{+{ }^{\prime}}(0)-P_{2 i-1}^{+^{\prime}}(0) .
$$

Hence

$$
\sum_{i=1}^{N} m_{i}(0)=P_{-1}^{+^{\prime}}(0)-P_{1}^{+^{\prime}}(0)+\cdots+P_{2 N-3}^{+^{\prime}}(0)-P_{2 N-1}^{+^{\prime}}(0)=-P_{2 N-1}^{+{ }^{\prime}}(0)
$$

This proves $(3.11)$

3.2. The class $\mathcal{U}_{\kappa}^{k}(J)$ and linear fractional transformations. Let $J$ and $\mathcal{Z}$ be the $2 \times 2$ matrices

$$
J=\left(\begin{array}{cc}
0 & -i \\
i & 0
\end{array}\right) \text { and } \mathcal{Z}=\left(\begin{array}{cc}
z & 0 \\
0 & 1
\end{array}\right) .
$$

Definition 3.6. Let $W(z)$ be a $2 \times 2$ matrix valued function meromorphic in $\mathbb{C}_{+}$and let $\mathfrak{h}_{W}^{+}$be the domain of holomorphy of $W$ in $\mathbb{C}_{+}, \kappa \in \mathbb{Z}_{+}$. Then $W(z)$ is called a generalized $J$-inner matrix valued function from the class $\mathcal{U}_{\kappa}(J)$, if

(i) the kernel

$$
\mathrm{K}_{\omega}^{W}(z)=\frac{J-W(z) J W(\omega)^{*}}{-i(z-\bar{\omega})}
$$

has $\kappa$ negative squares in $\mathfrak{h}_{W}^{+} \times \mathfrak{h}_{W}^{+}$;

(ii) $J-W(\mu) J W(\mu)^{*}=0$ for a.e. $\mu \in \mathbb{R}$.

A matrix valued function $W \in \mathcal{U}_{\kappa}(J)$ is said to belong to the class $\mathcal{U}_{\kappa}^{k}(J), \kappa, k \in \mathbb{Z}_{+}$, if

$$
\mathcal{Z} W \mathcal{Z}^{-1} \in \mathcal{U}_{k}(J)
$$

Consider the linear fractional transformation

$$
T_{W}[\tau]=\left(w_{11} \tau(z)+w_{12}\right)\left(w_{21} \tau(z)+w_{22}\right)^{-1}
$$

associated with the matrix valued function $W(z)=\left(w_{i, j}(z)\right)_{i, j=1}^{2}$. The linear fractional transformation associated with the product $W_{1} W_{2}$ of two matrix valued functions $W_{1}(z)$ and $W_{2}(z)$, coincides with the composition $T_{W_{1}} \circ T_{W_{2}}$.

The following statement is an easy corollary of Definition 3.6.

Lemma 3.7. Let $W \in \mathcal{U}_{\kappa_{1}}^{k_{1}}(J)$ and $\tau \in \mathbf{N}_{\kappa_{2}}^{k_{2}}, \kappa_{1}, \kappa_{2}, k_{1}, k_{2} \in \mathbb{Z}_{+}$. Then $T_{W}[\tau] \in \mathbf{N}_{\kappa^{\prime}}^{k^{\prime}}$, where $\kappa^{\prime} \leq \kappa_{1}+\kappa_{2}, k^{\prime} \leq k_{1}+k_{2}$. 
Proof. The proof of the inclusion $T_{W}[\tau] \in \mathbf{N}_{\kappa^{\prime}}$ for some $\kappa^{\prime} \leq \kappa_{1}+\kappa_{2}$ is similar to that in [13, Lemma 3.4].

Let us denote $f(z)=T_{W}(z)[\tau(z)]$. Then

$$
z f(z)=T_{\mathcal{Z} W(z) \mathcal{Z}^{-1}}[z \tau(z)] .
$$

Since $\mathcal{Z} W \mathcal{Z}^{-1} \in \mathcal{U}_{k_{1}}$ and $z \tau \in \mathbf{N}_{k_{2}}$ then $f \in \mathbf{N}_{k^{\prime}}$ for some $k^{\prime} \leq k_{1}+k_{2}$. This proves that $T_{W}[\tau] \in \mathbf{N}_{\kappa^{\prime}}^{k^{\prime}}$ for some $\kappa^{\prime} \leq \kappa_{1}+\kappa_{2}, k^{\prime} \leq k_{1}+k_{2}$.

In the present paper two special types of matrix valued functions from $\mathcal{U}_{\kappa}^{k}(J)$ play an important role (see [17, Lemma 2.11, Lemma 2.12]).

Lemma 3.8. Let $m(z)$ be a real polynomial such that $\kappa_{-}(z m(z))=\kappa_{1}, \kappa_{-}(m(z))=k_{1}$, let $M(z)$ be a $2 \times 2$ matrix valued function

$$
M(z)=\left(\begin{array}{cc}
1 & 0 \\
-z m(z) & 1
\end{array}\right)
$$

and let $\tau$ be a meromorphic function, such that

$$
\tau(z)^{-1}=o(z) \quad \text { as } \quad z \widehat{\rightarrow} \infty .
$$

Then $M \in \mathcal{U}_{\kappa_{1}}^{k_{1}}(J)$ and the following equivalences hold:

$$
\begin{aligned}
& \tau \in \mathbf{N}_{\kappa_{2}} \Longleftrightarrow T_{M}[\tau] \in \mathbf{N}_{\kappa_{1}+\kappa_{2}}, \\
& \tau \in \mathbf{N}_{\kappa_{2}}^{k_{2}} \Longleftrightarrow T_{M}[\tau] \in \mathbf{N}_{\kappa_{1}+\kappa_{2}}^{k_{1}+k_{2}} .
\end{aligned}
$$

Lemma 3.9. Let $l(z)$ be a real polynomial such that $\kappa_{-}(l(z))=\kappa_{1}, \kappa_{-}(z l(z))=k_{1}$, let $L(z)$ be the $2 \times 2$ matrix valued function

$$
L(z)=\left(\begin{array}{cc}
1 & l(z) \\
0 & 1
\end{array}\right)
$$

and let $\phi$ be a meromorphic function, such that

$$
\phi(z)=o(1) \quad \text { as } \quad z \widehat{\rightarrow} \infty .
$$

Then $L \in \mathcal{U}_{\kappa_{1}}^{k_{1}}(J)$ and the following equivalences hold:

$$
\begin{aligned}
& \phi \in \mathbf{N}_{\kappa_{2}} \Longleftrightarrow T_{L}[\phi] \in \mathbf{N}_{\kappa_{1}+\kappa_{2}}, \\
& \phi \in \mathbf{N}_{\kappa_{2}}^{k_{2}} \Longleftrightarrow T_{L}[\phi] \in \mathbf{N}_{\kappa_{1}+\kappa_{2}}^{k_{1}+k_{2}} .
\end{aligned}
$$

3.3. Truncated indefinite moment problem and resolvent matrices. As was mentioned in Theorem 1.2 the matrix valued function $W_{2 N}(z)$ defined by (1.26) is the resolvent matrix of a truncated even indefinite moment problem $M P_{\kappa}^{k}(\mathbf{s}, 2 N-1)$ with $\kappa \geq \kappa_{N}$ and $k \geq k_{N}^{+}$, where $\kappa_{N}$ and $k_{N}^{+}$are defined by (1.18). The resolvent matrix $W_{2 N}(z)$ provides an example of generalized $J$-inner matrix valued function from the class $\mathcal{U}_{\kappa_{N}}^{k_{N}^{+}}(J)$.

Theorem 3.10. ([17]) Let $\mathbf{s}=\left\{s_{i}\right\}_{i=0}^{\infty} \in \mathcal{H}_{\kappa}^{k, r e g}$, let $P_{j}^{+}(z)$ and $Q_{j}^{+}(z)$ be generalized Stieltjes polynomials of the first and second kind, let $m_{i}(z), l_{i}$ be defined by (2.15) and (2.16) and let $W_{2 N}(z), M_{i}(z)$ and $L_{i}$ be given by (1.26) and (1.28). Then

(i) The matrix valued function $W_{2 N}(z)$ admits the factorization

$$
W_{2 N}(z)=M_{1}(z) L_{1} \ldots M_{N}(z) L_{N} ;
$$

(ii) $W_{2 N} \in \mathcal{U}_{\kappa_{N}}^{k_{N}^{+}}(J)$. 
Proof. It follows from (3.1) that

$$
W_{2 N}(z)=W_{2 N-2}(z) M_{N}(z) L_{N} .
$$

Applying (3.25) $N$ times one obtains (3.24).

By [17, Theorem $4.3(4)]$

$$
\kappa_{N}=\sum_{j=1}^{N} \kappa_{-}\left(z m_{j}\right), \quad k_{N}^{+}=\sum_{j=1}^{N} k_{-}\left(m_{j}\right)+\sum_{j=1}^{N} \kappa_{-}\left(z l_{j}\right) .
$$

Since by Lemmas 3.8, $3.9 M_{i} \in \mathcal{U}_{\kappa_{-}\left(z m_{i}\right)}^{\kappa_{-}\left(m_{i}\right)}, L_{i} \in \mathcal{U}_{0}^{\kappa_{-}\left(z l_{i}\right)}, i \in \mathbb{N}$, one obtains from the factorization formula $(3.24) W_{2 N} \in \mathcal{U}_{\kappa^{\prime}}^{k^{\prime}}(J)$, where

$$
\kappa^{\prime} \leq \kappa_{N}, \quad k^{\prime} \leq k_{N}^{+}
$$

On the other hand it follows from Lemma 3.7 that $f:=T_{W_{2 N}[0]} \in \mathbf{N}_{\kappa_{N}}^{k_{N}^{+}}$. Therefore, by Lemma 3.7

$$
\kappa_{N} \leq \kappa^{\prime}, \quad k_{N}^{+} \leq k^{\prime}
$$

The statement (ii) follows from (3.26) and (3.27).

Let us formulate an analog of Theorems 1.2 and 3.10 for the odd truncated indefinite moment problem (see [17]).

Theorem 3.11. Let $\mathbf{s}=\left\{s_{i}\right\}_{i=0}^{2 n_{N}-2} \in \mathcal{H}_{\kappa}^{k, r e g}$, let $P_{j}^{+}(z)$ and $Q_{j}^{+}(z)(0 \leq j \leq 2 N-1)$ be generalized Stieltjes polynomials, let $M_{i}(z)$ and $L_{i}$ be given by (1.28) and let the matrix valued function $W_{2 N-1}$ be defined by

$$
W_{2 N-1}(z)=\left(\begin{array}{ll}
Q_{2 N-1}^{+}(z) & Q_{2 N-2}^{+}(z) \\
P_{2 N-1}^{+}(z) & P_{2 N-2}^{+}(z)
\end{array}\right) .
$$

Then

(i) The odd moment problem $M P_{\kappa}^{k}\left(s, 2 n_{N}-2\right)$ is solvable, if and only if

$$
\kappa_{N}:=\nu_{-}\left(S_{n_{N}}\right) \leq \kappa \quad \text { and } \quad k_{N}:=\nu_{-}\left(S_{n_{N}-1}^{+}\right) \leq k .
$$

(ii) $f \in \mathcal{M}_{\kappa}^{k}\left(s, 2 n_{N}-2\right)$ if and only if $f$ admits the representation

$$
f(z)=T_{W_{2 N-1}(z)}[\tau(z)]=\frac{Q_{2 N-1}^{+}(z) \tau(z)+Q_{2 N-2}^{+}(z)}{P_{2 N-1}^{+}(z) \tau(z)+P_{2 N-2}^{+}(z)},
$$

where $\tau \in N_{\kappa-\kappa_{N}}^{k-k_{N}}$ and $\tau(z)^{-1}=o(z)$ as $z \widehat{\rightarrow} \infty$.

(iii) The matrix valued function $W_{2 N-1}(z)$ belongs to the class $\mathcal{U}_{\kappa_{N}}^{k_{N}}(J)$ and admits the factorization

$$
W_{2 N-1}(z)=M_{1}(z) L_{1} \ldots L_{N-1} M_{N}(z) .
$$

Substituting in (3.30) $\tau(z)=\infty$ one obtains from Theorem 3.11 the following:

Corollary 3.12. The function $\frac{Q_{2 N-1}^{+}(z)}{P_{2 N-1}^{+}(z)}$ belongs to the class $\mathbf{N}_{\kappa_{N}}^{k_{N}}$.

Similarly, substituting in (1.23) $\tau(z) \equiv 0$ one obtains from Theorem 1.2.

Corollary 3.13. The function $\frac{Q_{2 N}^{+}(z)}{P_{2 N}^{+}(z)}$ belongs to the class $\mathbf{N}_{\kappa_{N}}^{k^{+}}$. 
3.4. Two lemmas about linear fractional transformations $T_{M}$ and $T_{L}$. The statements of Lemmas 3.8 and 3.9 fail to hold if the conditions

$$
\begin{gathered}
\tau(z)^{-1}=o(z) \quad \text { as } \quad z \widehat{\rightarrow} \infty, \\
\phi(z)=o(1) \text { as } \quad z \widehat{\rightarrow} \infty
\end{gathered}
$$

are not satisfied. In these cases the number of moments interpolating by the linear fractional transformations $T_{W}[\tau]$ can be reduced and also their indices can decrease. We will start with the linear fractional transformations $T_{M}$ in the simplest cases when $m$ is a positive constant and hence $M \in \mathcal{U}_{0}^{0}(J)$.

Lemma 3.14. Let $M(z)$ be a $2 \times 2$ matrix valued function

$$
M(z)=\left(\begin{array}{cc}
1 & 0 \\
-z m & 1
\end{array}\right), \quad m>0,
$$

let $\tau \in \mathbf{N}_{\kappa}^{k}$ be a function from $\mathbf{N}_{\kappa}^{k}$, such that $\tau(z)^{-1} \neq o(z)$ as $z \widehat{\rightarrow} \infty$ and let $\phi=T_{M}[\tau]$. Then

(i) either $\phi \in \mathbf{N}_{\kappa}^{k}$ and $\phi(z)=o(1)$ as $z \widehat{\rightarrow} \infty$,

(ii) or $\phi \in \mathbf{N}_{\kappa-1}^{k}$.

Proof. 1. Verification of (i) for $\tau \in \mathbf{N}_{\kappa}^{k}$, such that:

$$
\lim _{z \rightarrow \infty} \frac{-1}{z \tau(z)}=-\infty
$$

By Lemma $2.1-\tau^{-1} \in \mathbf{N}_{\kappa}^{-k}$. If (3.35) holds then $m z-\tau(z)^{-1}$ has GPNT (generalized pole of negative type) at $\infty$ of the same multiplicity as $-\tau(z)^{-1}$, i.e.

$$
\kappa_{\infty}\left(m z-\frac{1}{\tau(z)}\right)=\kappa_{\infty}\left(-\frac{1}{\tau(z)}\right) .
$$

Since also

$$
\kappa_{\infty}\left(m-\frac{1}{z \tau(z)}\right)=\kappa_{\infty}\left(-\frac{1}{z \tau(z)}\right),
$$

one obtains by Theorem $2.2 m z-\tau(z)^{-1} \in \mathbf{N}_{\kappa}^{-k}$. Hence $\phi(z)=\frac{-1}{m z-\tau(z)^{-1}} \in \mathbf{N}_{\kappa}^{k}$ by Lemma 2.1 and, moreover, $\phi(z)=o(1)$ as $z \widehat{\rightarrow} \infty$, since

$$
\lim _{z \rightarrow \infty} \phi(z)=\lim _{z \rightarrow \infty} \frac{1 / z}{m-(z \tau(z))^{-1}}=0 .
$$

2. Verification of (i) for $\tau \in \mathbf{N}_{\kappa}^{k}$, such that:

$$
\lim _{z \rightrightarrows \infty} \frac{-1}{z \tau(z)}=a
$$

and $a \geq 0$. In this case $\kappa_{\infty}\left(-\tau(z)^{-1}\right)=0$ and $-\tau(z)^{-1}$ admits the representation

$$
-\tau(z)^{-1}=a z-\tau_{1}(z)^{-1},
$$

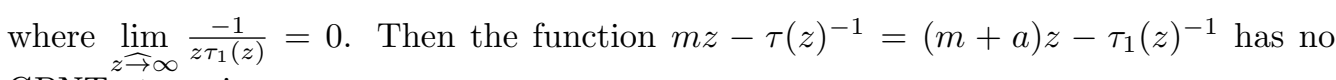
GPNT at $\infty$ since

$$
\lim _{z \rightarrow \infty}\left(m-\frac{1}{z \tau(z)}\right)=m+a>0 .
$$

Then (3.36) and (3.37) hold and by Theorem $2.2 m z-\tau(z)^{-1} \in \mathbf{N}_{\kappa}^{-k}$. Moreover,

$$
\lim _{z \rightarrow \infty} \phi(z)=\lim _{z \rightarrow \infty} \frac{1 / z}{m+a-(z \tau(z))^{-1}}=0 .
$$

and hence (i) holds. 
3. Verification of (i) for $\tau \in \mathbf{N}_{\kappa}^{k}$, such that (3.38) holds and $a<-m$ : In this case

$$
\lim _{z \rightarrow \infty}\left(m-\frac{1}{z \tau(z)}\right)=m+a<0
$$

and hence

$$
\kappa_{\infty}\left(m z-\frac{1}{\tau(z)}\right)=\kappa_{\infty}\left(-\frac{1}{\tau(z)}\right)=1 .
$$

Since also (3.37) holds then by Theorem $2.2 m z-\tau(z)^{-1} \in \mathbf{N}_{\kappa}^{-k}$, and by (3.40) $\phi(z)=$ $o(1)$ as $z \widehat{\rightarrow} \infty$. Hence (i) holds.

4. Verification of (ii) for $\tau \in \mathbf{N}_{\kappa}^{k}$, such that (3.38) holds and $a \in[-m, 0)$ : In this case

$$
\kappa_{\infty}\left(m z-\frac{1}{\tau(z)}\right)=0, \quad \kappa_{\infty}\left(-\frac{1}{\tau(z)}\right)=1
$$

and since (3.37) holds then $m z-\tau(z)^{-1} \in \mathbf{N}_{\kappa-1}^{-k}$ by Theorem 2.2. Therefore, $\phi(z)=$ $\frac{-1}{m z-\tau(z)^{-1}} \in \mathbf{N}_{\kappa-1}^{k}$ by Lemma 2.1. This proves (ii).

Lemma 3.15. Let $L$ be the $2 \times 2$ matrix

$$
L=\left(\begin{array}{ll}
1 & l \\
0 & 1
\end{array}\right), \quad \text { where } l>0,
$$

let $\phi$ be a function from $\mathbf{N}_{\kappa}^{k}$, such that

$$
\phi(z) \neq o(1) \quad \text { as } \quad z \widehat{\rightarrow} \infty
$$

and let $\tau=T_{L}[\phi]=l+\phi$. Then

(i) either $\tau \in \mathbf{N}_{\kappa}^{k}$ and $\tau(z)^{-1}=o(z)$ as $z \widehat{\rightarrow} \infty$,

(ii) or $\tau \in \mathbf{N}_{\kappa}^{k-1}$.

Proof. 1. Verification of $(i)$ for $\phi \in \mathbf{N}_{\kappa}^{k}$, such that $z \phi(z)$ has a GPNT at $\infty$ and:

$$
\lim _{z \rightarrow \infty} \phi(z)=-\infty \text {. }
$$

The function $z \tau(z)=l z+z \phi(z)$ has GPNT at $\infty$ of the same multiplicity as $z \phi(z)$ and by Theorem $2.2 z \tau(z) \in \mathbf{N}_{k}$. Hence $\tau \in \mathbf{N}_{\kappa}^{k}$ and the equality

$$
\lim _{z \rightarrow \infty} \frac{1}{z \tau(z)}=\lim _{z \rightrightarrows \infty} \frac{1}{l z+z \phi(z)}=0
$$

implies that $\tau(z)^{-1}=o(z)$ as $z \widehat{\rightarrow} \infty$. Thus (1) holds.

2. Verification of $(i)$ for $\phi \in \mathbf{N}_{\kappa}^{k}$, such that $\lim _{z \rightarrow \infty} \phi(z)=a$ and $a>0$ : In this case $z \phi(z)$ admits the representation

$$
z \phi(z)=a z+z \phi_{1}(z)
$$

where $\lim _{z \rightarrow \infty} \phi_{1}(z)=0$ and $\phi_{1} \in \mathbf{N}_{\kappa}^{k}$ by Theorem 2.2. The function $z \tau(z)=l z+z \phi(z)$ has no GPNT at $\infty$ and therefore $\tau \in \mathbf{N}_{\kappa}^{k}$. Moreover,

$$
\lim _{z \leftrightharpoons \infty} \frac{1}{z \tau(z)}=\lim _{z \leftrightharpoons \rightarrow \infty} \frac{1}{(l+a) z+z \phi(z)}=0 .
$$

and hence $(i)$ holds.

3. If $\phi \in \mathbf{N}_{\kappa}^{k}, \lim _{z \rightarrow \infty} \phi(z)=a$ and $a<-l$, then also $(i)$ holds: In this case

$$
\kappa_{\infty}(z \phi)=1 \quad \text { and } \quad \kappa_{\infty}(z \tau)=1
$$

that is the functions $z \phi(z)$ and $z \tau(z)$ have GPNT at $\infty$ of the same multiplicity. By Theorem $2.2 \tau \in \mathbf{N}_{\kappa}^{k}$ and by (3.45) $\tau(z)^{-1}=o(z)$ as $z \widehat{\rightarrow} \infty$. Hence $(i)$ holds. 
4. If $\lim _{z \rightarrow \infty} \phi(z)=a$ and $a \in[-l, 0)$ then (ii) holds: In this case

$$
\kappa_{\infty}(z \phi)=1 \quad \text { and } \quad \kappa_{\infty}(z \tau)=0
$$

and hence $z \tau \in \mathbf{N}_{k-1}$ by Theorem 2.2. Thus $\tau \in \mathbf{N}_{\kappa}^{k-1}$.

Corollary 3.16. Let a matrix valued function $W_{2 n}(z)$ of the form (1.26) belong to the class $\mathcal{U}_{0}^{0}(J)$, let $\phi \in \mathbf{N}_{\kappa}^{k}$, (3.33) fails to hold, let $n \geq \min \{\kappa, k\}$ and let $f=T_{W_{2 n}}[\phi]$. Then there exists $r \in \mathbb{Z}_{+}(r \leq \min \{\kappa, k\})$, such that

(i) either $f \in \mathcal{M}_{\kappa-r}^{k-r}(\mathbf{s}, 2(n-r)-2)$;

(ii) or $f \in \mathcal{M}_{\kappa-r}^{k-r-1}(\mathbf{s}, 2(n-r)-3)$.

In particular, $f(z)=o(1)$ as $z \widehat{\rightarrow} \infty$.

Proof. By Theorem 3.11 the matrix valued function $W_{2 n}(z)$ admits the factorization

$$
W_{2 n}(z)=M_{1}(z) L_{1} \ldots M_{n}(z) L_{n} .
$$

Let us denote $\tau_{1}:=T_{L_{n}}[\phi]$. By Lemma 3.15 there are three possibilities

(a1) either $\tau_{1} \in \mathbf{N}_{\kappa}^{k}$ and $\tau_{1}^{-1}(z)=o(z)$;

(a2) or $\tau_{1} \in \mathbf{N}_{\kappa}^{k-1}$ and $\tau_{1}^{-1}(z)=o(z)$;

(a3) or $\tau_{1} \in \mathbf{N}_{\kappa}^{k-1}$ and $\tau_{1}^{-1}(z) \neq o(z)$.

In the case (a1) one obtains by Theorem $3.11 f \in \mathcal{M}_{\kappa}^{k}(\mathbf{s}, 2 n-2)$, which gives (i) with $r=0$.

In the case (a2) by Theorem $3.11 f \in \mathcal{M}_{\kappa}^{k-1}(\mathbf{s}, 2 n-2) \subset \mathcal{M}_{\kappa}^{k-1}(\mathbf{s}, 2 n-3)$, which gives (ii) with $r=0$.

In the case (a3) we apply Lemma 3.14 to the function $\phi_{1}(z)=T_{M_{n}}\left[\tau_{1}(z)\right]$ and then again there are three possibilities:

(b1) $\phi_{1}:=T_{M_{n}}[\tau] \in \mathbf{N}_{\kappa}^{k-1}$ and $\phi_{1}(z)=o(1)$;

(b2) $\phi_{1}:=T_{M_{n}}[\tau] \in \mathbf{N}_{\kappa-1}^{k-1}$ and $\phi_{1}(z)=o(1)$;

(b3) $\phi_{1}:=T_{M_{n}}[\tau] \in \mathbf{N}_{\kappa-1}^{k-1}$ and $\phi_{1}(z) \neq o(1)$.

In the case (b1) by Theorem 1.2 one obtains $f \in \mathcal{M}_{\kappa}^{k-1}(\mathbf{s}, 2 n-3)$ which gives (2) with $r=0$.

In the case (b2) by Theorem $1.2 f \in \mathcal{M}_{\kappa-1}^{k-1}(\mathbf{s}, 2 n-3) \subset \mathcal{M}_{\kappa-1}^{k-1}(\mathbf{s}, 2 n-4)$ which gives

(i) with $r=1$.

In the case (b3) one should continue this process based on Lemma 3.14, Lemma 3.15, Theorem 1.2 and Theorem 3.11.

If, for instance, $k \leq \kappa$ and if the process will not stop until the step $r=k$, then when applying Lemma 3.15 to $\phi_{k} \in \mathbf{N}_{\kappa-k}^{0}$ one obtains only one possibility: $\tau_{k+1} \in \mathbf{N}_{\kappa-k}^{0}$ and $\tau_{k+1}(z)^{-1}=o(z)$ as $z \widehat{\rightarrow} \infty$. Then the process stops and $f \in \mathcal{M}_{\kappa-k}^{0}(\mathbf{s}, 2(n-k)-2)$ by Theorem 3.11.

Similarly, one can treat the case $\kappa<k$ by using Lemma 3.14 and Theorem 1.2.

\section{Full indefinite moment PRoblem $M P_{\kappa}^{k}(\mathbf{s})$}

4.1. Indefinite moment problem $M P_{\kappa}^{k}(\mathbf{s})$ for $\mathbf{s} \in \mathcal{H}_{0}^{0}$. Recall, that the moment problem $M P_{\kappa}^{k}(\mathbf{s})$ is called indeterminate if it has more then one solution. A sequence $\mathbf{s}$ is called nondegenerate, if

there is $N \in \mathbb{N}, \quad$ such that $\quad D_{n} \neq 0, \quad D_{n}^{+} \neq 0$ for all $n \geq N$.

In this subsection we consider the indefinite moment problem $M P_{\kappa}^{k}(\mathbf{s})$ for a nondegenerate sequence $\mathbf{s} \in \mathcal{H}_{0}^{0}$. As is known, see [1, Theorem 0.5$]$, for a nondegenerate sequence $\mathbf{s} \in \mathcal{H}_{0}^{0}$ the corresponding moment problem $M P_{0}^{0}(\mathbf{s})$ is indeterminate, if and only if

$$
\sum_{i=1}^{\infty} m_{i}<\infty \text { and } \sum_{i=1}^{\infty} l_{i}<\infty .
$$


Theorem 4.1. Let $s$ be a nondegenerate sequence from $\mathcal{H}_{0}^{0}$ and let (4.2) holds. Then

(i) The moment problem $M P_{\kappa}^{k}(s)$ is solvable and indeterminate for any pair of $\kappa, k \in$ $\mathbb{Z}_{+}$.

(ii) The sequence of resolvent matrices $W_{2 n}(z)$ converges to an entire matrix valued function $W_{\infty}^{+}(z)=\left(w_{i j}^{+}(z)\right)_{i, j=1}^{2}$ of order $\leq 1 / 2$.

(iii) The formula

$$
f(z)=\frac{w_{11}^{+}(z) \tau(z)+w_{12}^{+}(z)}{w_{21}^{+}(z) \tau(z)+w_{22}^{+}(z)}
$$

establishes a one-to-one correspondence between the class $\mathcal{M}_{\kappa}^{k}(\boldsymbol{s})$ and the set of functions $\tau \in \mathbf{N}_{\kappa}^{k}$.

Proof. 1. Verification of (ii): Due to [27, Appendix II.13] the Stieltjes moment problem $\mathcal{M}_{0}^{0}\left(\mathbf{s}^{(N)}\right)$ is indeterminate if and only if (4.2) holds and in this case the sequence $W_{2 N}(z)$ (see (1.26)) of its resolvent matrices converges to an entire matrix valued function $W_{\infty}^{+}(z)=\left(w_{i j}^{+}(z)\right)_{i, j=1}^{2}$ of order $\leq 1 / 2$.

2. Verification of the implication $f \in \mathcal{M}_{\kappa}^{k}(s) \Longrightarrow \tau \in \mathbf{N}_{\kappa}^{k}$ : Since $f \in \mathcal{M}_{\kappa}^{k}(\mathbf{s})$ then $f \in \mathcal{M}_{\kappa}^{k}(\mathbf{s}, 2 n-1)$ for every $n \in \mathbb{N}$. By Theorem 1.2 there exists a sequence of functions $\phi_{N}(z) \in \mathbf{N}_{\kappa}^{k}$ such that (3.33) holds and

$$
f(z)=T_{W_{2 N}(z)}\left[\phi_{N}(z)\right] \text { for every } \quad N \in \mathbb{N} .
$$

It follows from (4.4) that

$$
-\left(Q_{2 N-1}^{+}(z)-f(z) P_{2 N-1}^{+}(z)\right) \phi_{N}(z)=Q_{2 N}^{+}(z)-f(z) P_{2 N}^{+}(z) .
$$

Notice that $Q_{2 N-1}^{+}(z)-f(z) P_{2 N-1}^{+}(z) \not \equiv 0$, since otherwise we would have $Q_{2 N}^{+}(z)-$ $f(z) P_{2 N}^{+}(z) \equiv 0$, which contradicts the generalized Liouville-Ostrogradsky identity (3.7).

As was mentioned above the matrix valued functions $W_{2 N}(z)$ converges locally uniformly in $\mathbb{C}$ to $W_{\infty}^{+}(z)=\left(w_{i j}^{+}(z)\right)_{i, j=1}^{2}$, in particular,

$$
w_{11}^{+}(z)=\lim _{N \rightarrow \infty} Q_{2 N-1}^{+}(z), \quad w_{21}^{+}(z)=\lim _{N \rightarrow \infty} P_{2 N-1}^{+}(z) .
$$

By Corollary $3.12 \frac{Q_{2 N-1}^{+}}{P_{2 N-1}^{+}} \in \mathbf{N}_{0}^{0}=S$. Hence the limiting function $\frac{w_{11}^{+}(z)}{w_{21}^{+}(z)}$ belongs to $\mathbf{N}_{0}^{0}=S$ and it corresponds to $\phi=\infty$ in the linear fractional transformation $f=T_{W_{\infty}^{+}}[\tau]$. Apart from this case the function

$$
w_{11}^{+}(z)-f(z) w_{21}^{+}(z)=\lim _{N \rightarrow \infty}\left(Q_{2 N-1}^{+}(z)-f(z) P_{2 N-1}^{+}(z)\right)
$$

is not identically equal to 0 . Let $\Omega$ be the open set of points in $\mathbb{C}_{+}$such that $w_{11}^{+}(z)-$ $f(z) w_{21}^{+}(z) \neq 0$. Then for every point $z \in \Omega$ the sequence of functions

$$
\phi_{N}(z):=\frac{Q_{2 N}^{+}(z)-f(z) P_{2 N}^{+}(z)}{Q_{2 N-1}^{+}(z)-f(z) P_{2 N-1}^{+}(z)}
$$

is correctly defined in a neighborhood of $z$ and converges locally uniformly in $\Omega$ to a function $\phi(z)$. Since $\phi_{N} \in \mathbf{N}_{\kappa}^{k}$ then $\phi \in \mathbf{N}_{\kappa^{\prime}}^{k^{\prime}}$ with $\kappa^{\prime} \leq \kappa$ and $k^{\prime} \leq k$. It follows from (4.4) that

$$
f(z)=T_{W_{\infty}^{+}(z)}[\phi(z)]
$$

and by Lemma $3.7 f \in \mathbf{N}_{\kappa^{\prime \prime}}^{k^{\prime \prime}}$ with $\kappa^{\prime \prime} \leq \kappa^{\prime} \leq \kappa$ and $k^{\prime \prime} \leq k^{\prime} \leq k$. Since $f \in \mathbf{N}_{\kappa}^{k}$ this implies $\kappa^{\prime \prime}=\kappa^{\prime}=\kappa$ and $k^{\prime \prime}=k^{\prime}=k$ and hence $\tau \in \mathbf{N}_{\kappa}^{k}$.

3. Proof of the fact that $T_{W_{\infty}^{+}}[\tau]$ satisfies (3.33) for every $\tau \in \mathbf{N}_{\kappa}^{k}, \kappa, k \in \mathbb{Z}_{+}$. Application of the Schur algorithm to the Stieltjes moment problem $\mathcal{M}_{\kappa}^{k}(\mathbf{s})$ gives on the $N$-th step an induced sequence $\mathbf{s}^{(N)}$, which can be found as the sequence of coefficients 
of the series expansion $-\sum_{i=0}^{\infty} s_{i}^{(N)} z^{-(i+1)}$ corresponding to the continued fraction (2.17). Denote by $W_{2 j}^{(N)}(z)$ the resolvent matrix of the corresponding Stieltjes moment problem $\mathcal{M}_{0}^{0}\left(\mathbf{s}^{(N)}, 2 j-1\right)$.

$$
W_{2 j}^{(N)}(z)=\left(\begin{array}{ll}
Q_{2 j-1}^{N,+}(z) & Q_{2 j}^{N,+}(z) \\
P_{2 j-1}^{N,+}(z) & P_{2 j}^{N,+}(z)
\end{array}\right), \quad j \in \mathbb{N} .
$$

Then for every $j \in \mathbb{N}$ the resolvent matrix $W_{2 j}(z)$ admits the factorization (see [17, Proposition 4.7])

$$
W_{2 j}(z)=W_{2 N}(z) W_{2(j-N)}^{(N)}(z) .
$$

Taking the limit as $j \rightarrow \infty$ one obtains the following factorization of the entire matrix valued function $W_{\infty}^{+}(z)$

$$
W_{\infty}^{+}(z)=W_{2 N}(z) W_{\infty}^{(N)}(z),
$$

where $W_{\infty}^{(N)}(z)$ is the resolvent matrix of the induced Stieltjes moment problem $\mathcal{M}_{0}^{0}\left(\mathbf{s}^{(N)}\right)$. Then $f=T_{W_{\infty}^{+}}[\tau]$ admits the representation

$$
f=T_{W_{2 N}}\left[\phi_{N}\right], \quad \text { where } \quad \phi_{N}=T_{W_{\infty}^{(N)}}[\tau] \in \mathbf{N}_{\kappa^{\prime}}^{k^{\prime}}, \quad \text { with } \kappa^{\prime} \leq \kappa \text { and } k^{\prime} \leq k .
$$

Since $W_{2 N} \in \mathcal{U}_{0}^{0}$ by Corollary 3.16 one obtains $f=o(1)$, if $N>\min \{\kappa, k\}$.

4. Verification of the implication $\tau \in \mathbf{N}_{\kappa}^{k} \Longrightarrow f=T_{W_{\infty}^{+}}[\tau] \in \mathcal{M}_{\kappa}^{k}(\boldsymbol{s})$ : Due to the above item 3 the function $\phi_{N}$ from (4.10) satisfies the condition (3.33).

Next By Theorem $1.2 f \in \mathcal{M}_{\kappa^{\prime}}^{k^{\prime}}(\mathbf{s}, 2 N-1)$. Since $N$ can be chosen arbitrarily large $f \in \mathcal{M}_{\kappa^{\prime}}^{k^{\prime}}(\mathbf{s})$. Now it follows from the item $\mathbf{2}$ that $\tau \in \mathbf{N}_{\kappa^{\prime}}^{k^{\prime}}$ and hence $\kappa^{\prime}=\kappa$ and $k^{\prime}=k$.

5. Verification of (i): Solvability of the indefinite Stieltjes moment problem $M P_{\kappa}^{k}(\mathbf{s})$ for any pair of $\kappa, k \in \mathbb{Z}_{+}$follows from the item 4. The formula (4.3) gives two different solutions of the problem $M P_{\kappa}^{k}(\mathbf{s})$ for different parameter functions $\tau_{1}, \tau_{2} \in \mathbf{N}_{\kappa}^{k}$ and thus the problem $M P_{\kappa}^{k}(\mathbf{s})$ is indeterminate.

\subsection{Indefinite moment problem $M P_{\kappa}^{k}(\mathbf{s})$, general case.}

Theorem 4.2. Let $s$ be a nondegenerate sequence from $\mathcal{H}_{\kappa_{0}}^{k_{0}, \text { reg }}, \kappa_{0}, k_{0} \in \mathbb{N}$ and let $l_{j}$ and $m_{j}(z)(j \in \mathbb{N})$ be parameters of the generalized $\mathbf{S}$-fraction (2.12). Then the moment problem $M P_{\kappa_{0}}^{k_{0}}(\boldsymbol{s})$ is indeterminate, if and only if

$$
\sum_{i=1}^{\infty} m_{i}(0)<\infty \quad \text { and } \quad \sum_{i=1}^{\infty} l_{i}<\infty
$$

If (4.11) holds, then

(i) The sequence of resolvent matrices $W_{2 n}(z)$ converges to an entire matrix valued function $W_{\infty}^{+}(z)=\left(w_{i j}^{+}(z)\right)_{i, j=1}^{2}$ of order $\leq 1 / 2$.

(ii) The moment problem $M P_{\kappa}^{k}(s)$ is solvable, if and only if

$$
\kappa_{0} \leq \kappa, \text { and } k_{0} \leq k .
$$

(iii) The formula

$$
f(z)=\frac{w_{11}^{+}(z) \tau(z)+w_{12}^{+}(z)}{w_{21}^{+}(z) \tau(z)+w_{22}^{+}(z)}
$$

establishes a one-to-one correspondence between the class $\mathcal{M}_{\kappa}^{k}(s)$ and the set of functions $\tau \in \mathbf{N}_{\kappa-\kappa_{0}}^{k-k_{0}}$. 
Proof. 1. Redaction of the indefinite moment problem $M P_{\kappa}^{k}(s)$ to a classical one: Let us choose $N$ big enough, so that

$$
\nu_{-}\left(S_{j}\right)=\kappa_{0}=\nu_{-}\left(S_{n_{N}}\right), \quad \nu_{-}\left(S_{j}^{(1)}\right)=k_{0}=\nu_{-}\left(S_{n_{N}}^{(1)}\right) \quad \text { for all } \quad j \geq n_{N} .
$$

Then the induced sequence $\mathbf{s}^{(N)}$ which arises on the $N$-th step of the Schur algorithm (see Section 2.2) belongs to the class $\mathcal{H}_{0}^{0}$. The corresponding Stieltjes moment problem $\mathcal{M}_{0}^{0}\left(\mathbf{s}^{(N)}\right)$ is classical. By Lemma 2.4

$$
f \in \mathcal{M}_{\kappa}^{k}(\mathbf{s}) \Longleftrightarrow f_{N}=T_{W_{2 n_{N}}}[f] \in \mathcal{M}_{\kappa-\kappa_{0}}^{k-k_{0}}\left(\mathbf{s}^{(N)}\right) .
$$

In particular,

$$
f \in \mathcal{M}_{\kappa_{0}}^{k_{0}}(\mathbf{s}) \Longleftrightarrow f_{N}=T_{W_{2 n_{N}}}[f] \in \mathcal{M}_{0}^{0}\left(\mathbf{s}^{(N)}\right) .
$$

Hence the problem $\mathcal{M}_{\kappa_{0}}^{k_{0}}\left(\mathbf{s}^{(N)}\right)$ is indeterminate if and only if the Stieltjes moment problem $\mathcal{M}_{0}^{0}\left(\mathbf{s}^{(N)}\right)$ is indeterminate. The latter is equivalent to (4.11).

2. Verification of (i): Let $P_{j}^{N,+}(z)$ and $Q_{j}^{N,+}(z)$ be generalized Stieltjes polynomials associated with the moment problem $\mathcal{M}_{0}^{0}\left(\mathbf{s}^{(N)}\right)$ and let $W_{2 j}^{(N)}$ (see (4.7)). If (4.11) holds, then the Stieltjes moment problem $\mathcal{M}_{0}^{0}\left(\mathbf{s}^{(N)}\right)$ is indeterminate and the sequence $W_{2 j}^{(N)}$ converges to an entire matrix valued function $W_{\infty}^{(N)}(z)=\left(w_{i j}^{(N)}(z)\right)_{i, j=1}^{2}$ of order $\leq 1 / 2$.

The resolvent matrices $W_{2 j}(z)$ of the indefinite moment problem $M P_{\kappa_{0}}^{k_{0}}(\mathbf{s}, 2 j-1)$ are connected with the resolvent matrices $W_{2(j-N)}^{(N)}(z)$ of the induced moment problem $M P_{0}^{0}\left(\mathbf{s}^{(N)}, 2(j-N)-1\right)$ by the formula (4.8). Therefore, the sequence of matrix valued functions $W_{2 n-1}(z)$ also converges to an entire matrix valued function $W_{\infty}^{+}(z)=$ $\left(w_{i j}^{+}(z)\right)_{i, j=1}^{2}$ of order $\leq 1 / 2$, which is connected with $W_{\infty}^{(N)}(z)$ by the formula (4.9).

3. Verification of (ii) and (iii) : By Proposition 2.1 (v) the inequalities (4.12) are necessary for solvability of the problem $\mathcal{M}_{\kappa}^{k}(\mathbf{s})$.

Now assume that (4.12) holds, let $\tau \in \mathbf{N}_{\kappa-\kappa_{0}}^{k-k_{0}}$ and let $f=T_{W_{\infty}^{+}}[\tau]$. Then by Theorem 4.1

$$
f_{N}=T_{W_{\infty}^{(N)}}[\tau] \in \mathcal{M}_{\kappa-\kappa_{0}}^{k-k_{0}}\left(\mathbf{s}^{(N)}\right)
$$

By (4.9) and Lemma 2.4

$$
f=T_{W_{2 N}}\left[f_{N}\right] \in \mathcal{M}_{\kappa}^{k}(\mathbf{s}) .
$$

Conversely, let $f \in \mathcal{M}_{\kappa}^{k}(\mathbf{s})$. Then by Lemma 2.4 there is a function $f_{N} \in \mathcal{M}_{\kappa-\kappa_{0}}^{k-k_{0}}\left(\mathbf{s}^{(N)}\right)$ such that $f=T_{W_{2 N}}\left[f_{N}\right]$. Hence by Theorem 4.1 there exists a function $\tau \in \mathbf{N}_{\kappa-\kappa_{0}}^{k-k_{0}}$, such that $f_{N}=T_{W_{\infty}^{(N)}}[\tau]$. Therefore, $f=T_{W_{\infty}^{+}}[\tau]$ for $\tau \in \mathbf{N}_{\kappa-\kappa_{0}}^{k-k_{0}}$.

Proposition 4.3. Let $s=\left\{s_{j}\right\}_{j=0}^{\infty} \in \mathcal{H}_{\kappa}^{k, r e g}$, let $P_{i}(z)$ and $Q_{i}(z)\left(i \in \mathbb{Z}_{+}\right)$be Lanczos polynomials of the first and second kind and let $l_{j}$ and $m_{j}(z)(j \in \mathbb{N})$ be parameters of the generalized $\mathbf{S}$-fraction (2.12). Assume additionally that

$$
l_{j}>0 \text { for all } j \in \mathbb{N} .
$$

Then the following statements are equivalent:

(i) the moment problem $M P_{\kappa}(s)$ is indeterminate;

(ii) the following series converge

$$
\sum_{i=0}^{\infty}\left|P_{i}(0)\right|^{2} \widetilde{b}_{i}^{-1}<\infty \quad \text { and } \quad \sum_{i=0}^{\infty}\left|Q_{i}(0)\right|^{2} \widetilde{b}_{i}^{-1}<\infty
$$


(iii) the following series converges

$$
\sum_{i=1}^{\infty}\left(l_{1}+l_{2}+\cdots+l_{i}\right)^{2} d_{i+1}(0)<\infty .
$$

Proof. By $[8,31]$ (i) and (ii) are equivalent.

Let us show, that (ii) and (iii) are equivalent. By the first equality in (3.10)

$$
Q_{i}(0)=-\left(l_{1}+l_{2}+\cdots+l_{i}\right) P_{i}(0) .
$$

and hence by (3.11)

$$
\sum_{i=0}^{N}\left|Q_{i}(0)\right|^{2} \widetilde{b}_{i}^{-1}=\sum_{i=0}^{N}\left(l_{1}+l_{2}+\cdots+l_{i}\right)^{2}\left|P_{i}(0)\right|^{2} \widetilde{b}_{i}^{-1}=\sum_{i=1}^{N}\left(l_{1}+l_{2}+\cdots+l_{i}\right)^{2} d_{i+1}(0) .
$$

This proves the implication (ii) $\Rightarrow$ (iii).

Conversely, if $\sum_{i=0}^{\infty}\left|Q_{i}(0)\right|^{2} \widetilde{b}_{i}^{-1}<\infty$, then the convergence of the series $\sum_{i=0}^{\infty}\left|P_{i}(0)\right|^{2} \widetilde{b}_{i}^{-1}$ follows from the inequality

$$
l_{1}^{2} \sum_{i=0}^{N}\left|P_{i}(0)\right|^{2} \widetilde{b}_{i}^{-1} \leq \sum_{i=0}^{N}\left(l_{1}+l_{2}+\cdots+l_{i}\right)^{2}\left|P_{i}(0)\right|^{2} \widetilde{b}_{i}^{-1}=\sum_{i=0}^{\infty}\left|Q_{i}(0)\right|^{2} \widetilde{b}_{i}^{-1} .
$$

This proves the implication (iii) $\Rightarrow$ (ii).

Remark 4.4. In the case $\kappa=k=0$ the above criterion for the Hamburger moment problem to be indeterminate is well known, see [1, Appendix, Theorem 0.5]. When treating system (3.1) as a Stieltjes string with masses $m_{j}$ and lengths $l_{j}$ one can consider series (4.21) as "the moment of inertia" of the string.

\section{PADÉ APPROXIMANTS}

Definition 5.1. ([3]) The $[L / M]$ Padé approximant for a formal power series

$$
-\sum_{j=0}^{\infty} \frac{s_{j}}{z^{j+1}}
$$

is a ratio

$$
f^{[L / M]}(z)=\frac{A^{[L / M]}(1 / z)}{B^{[L / M]}(1 / z)}
$$

of polynomials $A^{[L / M]}, B^{[L / M]}$ of formal degree $L, M$, respectively, such that $B^{[L / M]}(0) \neq$ 0 and

$$
f^{[L / M]}(z)+\sum_{j=0}^{L+M} \frac{s_{j}}{z^{j+1}}=O\left(\frac{1}{z^{L+M+1}}\right), \quad z \widehat{\rightarrow} \infty .
$$

The $[n / n]$ Padé approximant is called diagonal and the $[n / n-1]$ Padé approximant is called subdiagonal.

Remark 5.2. Notice, that for diagonal Padé approximants the representation (5.2) is equivalent to the representation

$$
f^{[n / n]}(z)=\frac{z^{n} A^{[n / n]}(1 / z)}{z^{n} B^{[n / n]}(1 / z)}
$$


as a ratio, where the numerator $z^{n} A^{[n / n]}(1 / z)$ is a polynomial of formal degree $n$ and the denominator $z^{n} B^{[n / n]}(1 / z)$ is a polynomial of exact degree $n$. For subdiagonal Padé approximants the representation (5.2) is equivalent to the representation

$$
f^{[n / n-1]}(z)=\frac{z^{n} A^{[n / n-1]}(1 / z)}{z^{n} B^{[n / n-1]}(1 / z)}
$$

as a ratio, where the numerator is a polynomial of formal degree $n$ and the denominator is a polynomial of exact degree $n$ vanishing at 0 .

Explicit formula for diagonal Padé approximants for sequences $\mathbf{s}=\left\{s_{j}\right\}_{j=0}^{\infty} \in \mathcal{H}_{\kappa}$ was found in [7], in the classical case $\mathbf{s} \in \mathcal{H}_{0}$ see [3, 38]. In this section we will formulate the corresponding statements for sequences $\mathbf{s}=\left\{s_{j}\right\}_{j=0}^{\infty} \in \mathcal{H}_{\kappa}^{k, \text { reg }}$.

Proposition 5.3. Let $s=\left\{s_{j}\right\}_{j=0}^{\infty} \in \mathcal{H}_{\kappa}^{k, \text { reg }}, \kappa, k \in \mathbb{Z}_{+}$. Then the $[n / n]$ Padé approximant for a formal power series (5.1) exists if $n \in \mathcal{N}(\mathbf{s})$ and

$$
f^{\left[n_{j} / n_{j}\right]}(z)=-\frac{Q_{j}(z)}{P_{j}(z)}=\frac{Q_{2 j}^{+}(z)}{P_{2 j}^{+}(z)}, \quad j \in \mathbb{N} .
$$

Proof. We present a proof of this statement from [17] for the convenience of the reader. It follows from (3.3) and Theorem 3.11 that the function

$$
-\frac{Q_{j}(z)}{P_{j}(z)}=\frac{Q_{2 j}^{+}(z)}{P_{2 j}^{+}(z)}=T_{W_{2 j}(z)}[0]
$$

belongs to $\mathcal{M}\left(\mathbf{s}, 2 n_{j}-1\right)$. Therefore, the function $-\frac{Q_{j}(z)}{P_{j}(z)}$ has the asymptotic

$$
-\frac{Q_{j}(z)}{P_{j}(z)}=-\frac{s_{0}}{z}-\cdots-\frac{s_{2 n_{j}-1}}{z^{2 n_{j}}}+O\left(\frac{1}{z^{2 n_{j}+1}}\right), \quad z \widehat{\rightarrow} \infty .
$$

Since $Q_{j}(z)$ is a polynomial of degree $n_{j}-n_{1}<n_{j}$ and $P_{j}(z)$ is a polynomial of exact degree $n_{j}$ the function $-\frac{Q_{j}(z)}{P_{j}(z)}$ is the $\left[n_{j} / n_{j}\right]$ Padé approximant for the formal power series (5.1) due to Remark 5.2 and (5.5).

In the following proposition stated in [16] without proof it is shown that the subdiagonal Padé approximants can be calculated in terms of generalized Stieltjes polynomials.

Proposition 5.4. Let $s=\left\{s_{j}\right\}_{j=0}^{\infty} \in \mathcal{H}_{\kappa}^{k, r e g}, \kappa, k \in \mathbb{Z}_{+}$. Then the $\left[n_{j} / n_{j}-1\right]$ Padé approximants for the formal power series (5.1) exists and has the form

$$
f^{\left[n_{j} / n_{j}-1\right]}(z)=\frac{Q_{2 j-1}^{+}(z)}{P_{2 j-1}^{+}(z)}, \quad j \in \mathbb{N} .
$$

Proof. It follows from (3.3) that

$$
\frac{Q_{2 j-1}^{+}(z)}{P_{2 j-1}^{+}(z)}=T_{W_{2 j-1}(z)}[\infty]
$$

By Theorem 3.11 the function $\frac{Q_{2 j-1}^{+}(z)}{P_{2 j-1}^{+}(z)}$ belongs to $\mathcal{M}\left(\mathbf{s}, 2 n_{j}-2\right)$, and hence it has the asymptotic

$$
\frac{Q_{2 j-1}^{+}(z)}{P_{2 j-1}^{+}(z)}=-\frac{s_{0}}{z}-\cdots-\frac{s_{2 n_{j}-2}}{z^{2 n_{j}-1}}+O\left(\frac{1}{z^{2 n_{j}+1}}\right), \quad z \widehat{\rightarrow} \infty .
$$


Since $Q_{2 j-1}^{+}(z)$ is a polynomial of degree $n_{j}-n_{1}<n_{j}$ and $P_{2 j-1}^{+}(z)$ is a polynomial of exact degree $n_{j}$ vanishing at 0 the function $-\frac{Q_{2 j-1}^{+}(z)}{P_{2 j-1}^{+}(z)}$ is the $\left[n_{j} / n_{j}-1\right]$ Padé approximant for the formal power series (5.1) due to Remark 5.2 and (5.7).

Lemma 5.5. Let $s=\left\{s_{j}\right\}_{j=0}^{\infty} \in \mathcal{H}_{\kappa}^{k, r e g}, \kappa, k \in \mathbb{Z}_{+}$, let $W_{2 N}(z)$ be given by $(1.26), N \in \mathbb{N}$ and let $\mathbf{s}^{(N)}$ be the induced sequence defined in Lemma 2.4, and let

$$
-\sum_{j=0}^{\infty} s_{j}^{(N)} z^{-(j+1)}
$$

be the corresponding formal power series. Then

(i) the diagonal Padé approximants for the formal power series (5.1) are connected with diagonal $g^{[n / n]}$ Padé approximants for the power series (5.8) by the formula

$$
f^{\left[n_{j} / n_{j}\right]}(z)=T_{W_{2 N}(z)}\left[g^{\left[n_{j}-n_{N} / n_{j}-n_{N}\right]}(z)\right], \quad j>N, \quad j \in \mathbb{N} .
$$

(ii) the subdiagonal Padé approximants for the power series (5.1) are connected with subdiagonal $g^{[n / n-1]}$ Padé approximants for the power series (5.8) by

$$
f^{\left[n_{j} / n_{j}-1\right]}(z)=T_{W_{2 N}(z)}\left[g^{\left[n_{j}-n_{N} / n_{j}-n_{N}-1\right]}(z)\right], \quad j>N, \quad j \in \mathbb{N} .
$$

Proof. Consider the induced moment problem $M P_{\kappa-\kappa_{N}}^{k-k_{N}^{+}}\left(\mathbf{s}^{(N)}, 2\left(n_{j}-n_{N}\right)-1\right)$ and let

$$
W_{2(j-N)}^{(N)}(z)=\left(\begin{array}{cc}
Q_{2(j-N)-1}^{N,+}(z) & Q_{2(j-N)}^{N,+}(z) \\
P_{2(j-N)-1}^{N,+}(z) & P_{2(j-N)}^{N,+}(z)
\end{array}\right)
$$

be the resolvent matrix of this moment problem. Then the matrices $W_{2 j}(z)$ and $W_{2(j-N)}^{(N)}(z)$ are connected by (see $\left.(4.8)\right)$

$$
W_{2 j}(z)=W_{2 N}(z) W_{2(j-N)}^{(N)}(z) .
$$

Similarly, the resolvent matrix

$$
W_{2(j-N)-1}^{(N)}(z)=\left(\begin{array}{ll}
Q_{2(j-N)-1}^{N,+}(z) & Q_{2(j-N)-2}^{N,+}(z) \\
P_{2(j-N)-1}^{N,+}(z) & P_{2(j-N)-2}^{N,+}(z)
\end{array}\right)
$$

of the moment problem $M P_{\kappa-\kappa_{N}}^{k-k_{N}}\left(\mathbf{s}^{(N)}, 2\left(n_{j}-n_{N}\right)-1\right)$ is connected with the matrix $W_{2 j-1}(z)$ by

$$
W_{2 j-1}(z)=W_{2 N}(z) W_{2(j-N)-1}^{(N)}(z)
$$

By Proposition 5.3 diagonal Padé approximants $g^{\left[n_{j}-n_{N} / n_{j}-n_{N}\right]}(z)$ for the formal power series (5.8) are given by

$$
g^{\left[n_{j}-n_{N} / n_{j}-n_{N}\right]}(z)=\frac{Q_{2\left(n_{j}-n_{N}\right)}^{N,+}(z)}{P_{2\left(n_{j}-n_{N}\right)}^{N,+}(z)}=T_{W_{2(j-N)}^{(N)}(z)}[0], \quad j \in \mathbb{N} .
$$

It follows from the factorization formula (5.12) and (5.15) that

$$
T_{W_{2 N}(z)}\left[g^{\left[n_{j}-n_{N} / n_{j}-n_{N}\right]}(z)\right]=T_{W_{2 j}(z)}[0] .
$$

Hence by Proposition $5.3 T_{W_{2 N}(z)}\left[g^{\left[n_{j}-n_{N} / n_{j}-n_{N}\right]}(z)\right]$ coincides with the diagonal Padé approximants $f^{\left[n_{j} / n_{j}\right]}(z)$ for the formal power series (5.1).

By Proposition 5.4 subdiagonal Padé approximants $g^{\left[n_{j}-n_{N} / n_{j}-n_{N}-1\right]}(z)$ for the formal power series (5.8) are given by

$$
g^{\left[n_{j}-n_{N} / n_{j}-n_{N}-1\right]}(z)=\frac{Q_{2\left(n_{j}-n_{N}\right)-1}^{N,+}(z)}{P_{2\left(n_{j}-n_{N}\right)-1}^{N,+}(z)}=T_{W_{2(j-N)-1}^{(N)}(z)}[\infty], \quad j \in \mathbb{N} .
$$


It follows from the factorization formula (5.14) and (5.16) that

$$
T_{W_{2 N}(z)}\left[g^{\left[n_{j}-n_{N} / n_{j}-n_{N}\right]}(z)\right]=T_{W_{2 j}(z)}[\infty] .
$$

Hence by Proposition $5.4 T_{W_{2 N}(z)}\left[g^{\left[n_{j}-n_{N} / n_{j}-n_{N}-1\right]}(z)\right]$ coincides with the subdiagonal Padé approximants $f^{\left[n_{j} / n_{j}-1\right]}(z)$ for the formal power series (5.1).

Theorem 5.6. Let $s=\left\{s_{j}\right\}_{j=0}^{\infty} \in \mathcal{H}_{\kappa}^{k, r e g}, \kappa, k \in \mathbb{Z}_{+}$. Then

(i) If the problem $M P_{\kappa}^{k}(\mathbf{s})$ is determinate, then diagonal and subdiagonal Padé approximants converge to the unique solution of $M P_{\kappa}^{k}(\mathbf{s})$ locally uniformly on $\mathbb{C} \backslash \mathbb{R}_{+}$.

(ii) If the problem $M P_{\kappa}^{k}(\mathbf{s})$ is indeterminate, then the sequence $f^{[n / n-1]}$ of subdiagonal Padé approximants converges locally uniformly on $\mathbb{C} \backslash \mathbb{R}_{+}$, while the sequence $f^{[n / n]}$ of diagonal Padé approximants is not convergent but precompact in the topology of locally uniform convergence.

Proof. Let us choose $N$ big enough, so that

$$
\nu_{-}\left(S_{j}\right)=\kappa=\nu_{-}\left(S_{n_{N}}\right), \quad \nu_{-}\left(S_{j}^{(1)}\right)=k=\nu_{-}\left(S_{n_{N}}^{(1)}\right) \quad \text { for all } \quad j \geq n_{N} .
$$

Then the induced sequence $\mathbf{s}^{(N)}$ belongs to the class $\mathcal{H}_{0}^{0}$ and by Lemma 5.5 the problem of convergence of diagonal and subdiagonal Padé approximants is reduced to the corresponding problem for diagonal and subdiagonal Padé approximants for the series $-\sum_{j=0}^{\infty} s_{j}^{(N)} z^{-(j+1)}$ corresponding to the classical Stieltjes moment problem $\mathcal{M}_{0}^{0}\left(\mathbf{s}^{(N)}\right)$. The stated results for classical Stieltjes moment problems were proved in [38, Theorems 5.30, 5.31].

\section{Example. Laguerre Polynomials}

The monic Laguerre polynomials $\widetilde{L}_{n}(z, \alpha):=(-1)^{n} z^{-\alpha} e^{z}\left(z^{\alpha+n} e^{-z}\right)^{(n)}$ are solutions of the three-term difference equation (see [39])

$$
y_{n+1}(z)+(2 n+\alpha+1-z) y_{n}(z)+(n+\alpha) n y_{n-1}(z), \quad n \in \mathbb{N} .
$$

subject to the initial conditions $\widetilde{L}_{-1}(z, \alpha) \equiv 0, \widetilde{L}_{0}(z, \alpha) \equiv 1$.

If $\alpha>-1$, then the polynomials $\left\{\widetilde{L}_{n}(z, \alpha)\right\}_{n=0}^{\infty}$ are orthogonal in the Hilbert space $L_{2}\left(\mathbb{R}_{+}, w_{\alpha}\right)$, with the weight function $w_{\alpha}(z)=z^{\alpha} e^{-z}$.

Here we consider the case when $\alpha<-1$ and $\alpha$ is not a negative integer. The case when $\alpha$ is a negative integer was treated in [35]. If $-k-1<\alpha<-k, k \in \mathbb{N}$, then $\widetilde{L}_{n}(z, \alpha)$ are orthogonal polynomials with respect to the indefinite inner product (see [11], [37])

$$
\langle f, g\rangle_{\alpha}=\int_{0}^{\infty} x^{\alpha}\left(e^{-x} f \bar{g}-\sum_{j=0}^{k-1}\left(e^{-x} f \bar{g}\right)^{(j)}(0) \frac{x^{j}}{j !}\right) d x .
$$

Polynomials $\widetilde{Q}_{n}(x, \alpha)$ of the second kind are defined as solutions of (6.1) subject to the initial conditions $\widetilde{Q}_{-1}(z, \alpha) \equiv-1$ and $\widetilde{Q}_{0}(z, \alpha) \equiv 0$.

The $P$ - fraction corresponding to the system (6.1) has the form (1.14) with the atoms $\left(a_{n}, b_{n}\right)$ given by

$$
b_{0}=\Gamma(1+\alpha), \quad b_{n}=n(n+\alpha) \quad \text { and } \quad a_{n-1}(z)=z-2 n-\alpha+1, \quad n \in \mathbb{N} .
$$

The moments $s_{n}$ for all $n \in \mathbb{N}$ are defined by

$$
s_{n}=\mathfrak{S}\left(z^{n}\right)=\Gamma(n+\alpha+1) .
$$


By the Buslaev formula, see [5, formula (13)] the determinants of $S_{n}$ and $S_{n}^{+}$take the form

$$
D_{n}=b_{0}^{n} b_{1}^{n-1} \ldots b_{n-1}=\prod_{j=1}^{n}(j-2) ! \Gamma(\alpha+j), \quad D_{n}^{+}=\prod_{j=1}^{n}(j-2) ! \Gamma(\alpha+j+1), \quad n \in \mathbb{N} .
$$

Therefore, the sequence $\mathbf{s}=\left\{s_{n}\right\}_{n=0}^{\infty}$ is regular and the set of its normal indices coincides with $\mathbb{N}$ and

$$
\mathbf{s} \in \mathcal{H}_{k}^{k-1}, \quad \text { if } \quad-2 k<\alpha<-2 k+1, \quad \mathbf{s} \in \mathcal{H}_{k}^{k}, \quad \text { if } \quad-2 k-1<\alpha<-2 k ;
$$

Next, it follows from (1.6) that the parameters of the generalized S-fraction take the form

$$
l_{n}:=\frac{D_{n}^{2}}{D_{n}^{+} D_{n-1}^{+}}=\frac{(n-1) ! \Gamma^{2}(1+\alpha)}{\Gamma(1+\alpha+n)}, \quad m_{n}:=\frac{\left(D_{n-1}^{+}\right)^{2}}{D_{n} D_{n-1}}=\frac{\Gamma(\alpha+n)}{(n-1) ! \Gamma^{2}(1+\alpha)}, \quad n \in \mathbb{N} .
$$

The monic Laguerre polynomials $\widetilde{L}_{n}(z, \alpha)$ can be calculated by (see [39], [41])

$$
\widetilde{L}_{n}(z, \alpha)=\sum_{k=0}^{n}\left(\begin{array}{l}
n \\
k
\end{array}\right) \frac{\Gamma(n+\alpha+1)}{\Gamma(k+\alpha+1)}(-1)^{n+k} z^{k} .
$$

Then using the formula

$$
Q_{n}(z, \alpha)=\mathfrak{S}_{t}\left(\frac{\widetilde{L}_{n}(z, \alpha)-\widetilde{L}_{n}(t, \alpha)}{z-t}\right)
$$

one can find the Lanczos polynomials of the second kind

$$
\begin{aligned}
Q_{n}(z, \alpha) & =\mathfrak{S}_{t}\left(\sum_{k=0}^{n}\left(\begin{array}{l}
n \\
k
\end{array}\right) \frac{\Gamma(n+\alpha+1)}{\Gamma(k+\alpha+1)}(-1)^{n+k} \frac{z^{k}-t^{k}}{z-t}\right)= \\
& =\sum_{k=1}^{n} z^{k-1} \Gamma(\alpha+n+1) \sum_{j=0}^{n-k}(-1)^{n+k+j}\left(\begin{array}{c}
n \\
k+j
\end{array}\right) \frac{\Gamma(\alpha+j+1)}{\Gamma(\alpha+k+j+1)} .
\end{aligned}
$$

Due to (3.3) the Stieltjes polynomials of the first and second kind are calculated by

$$
\begin{aligned}
& P_{2 n}^{+}(z, \alpha)=\Gamma(1+\alpha) \sum_{i=0}^{n}\left(\begin{array}{c}
n \\
i
\end{array}\right) \frac{(-1)^{i} z^{i}}{\Gamma(1+\alpha+i)} \\
& P_{2 n-1}^{+}(z, \alpha)=-\frac{1}{(n-1) !} \prod_{j=1}^{n}(j+n)\left(\sum_{i=0}^{n-1} \frac{(-1)^{i-1}(n-1) ! i z^{i}}{i !(n-i) ! \Gamma(1+\alpha+i)}+\frac{(-1)^{n-1} z^{n}}{\Gamma(1+\alpha+n)}\right) \\
& Q_{2 n}^{+}(z, \alpha)=\sum_{i=1}^{n} z^{i-1} \Gamma(1+\alpha) \sum_{j=0}^{n-i}(-1)^{i+j+1}\left(\begin{array}{c}
n \\
i+j
\end{array}\right) \frac{\Gamma(1+\alpha+n)}{\Gamma(1+\alpha+i+j+1)} \\
& Q_{2 n-1}^{+}(z, \alpha)=\Gamma(1+\alpha+n) \sum_{i=1}^{n} z^{i-1} \sum_{j=0}^{n-i} \frac{(-1)^{i+j-1} \Gamma(1+\alpha+j)(n-1) !}{i !(n-j-i) ! \Gamma(1+\alpha+i+j)}
\end{aligned}
$$

The set of solutions of the truncated moment problem $M P_{\kappa}^{k}(\mathbf{s}, 2 n-1)$ is described by the formula (1.23).

Notice that the full moment problem is determinate and in the case $\alpha>-1$ its unique solution of $M P_{0}^{0}(\mathbf{s})$ is given by

$$
f(z)=\int_{0}^{\infty} \frac{x^{\alpha} e^{-x}}{x-z} d x=z^{\frac{\alpha-1}{2}} e^{-\frac{z}{2}} W_{\frac{-\alpha-1}{2}, \frac{\alpha}{2}}(-z)
$$

where $W_{\alpha, \beta}$ is the Whittaker function, see [26, 9.222]. 
If $-2 k-1<\alpha<-2 k, k \in \mathbb{N}$, then the solution $f(z)$ of $M P_{k}^{k}(\mathbf{s})$ can be found by the formula

$$
f(z)=\mathfrak{S}_{x}\left(\frac{e^{-x} x^{\alpha}}{x-z}\right)=\int_{0}^{\infty} x^{\alpha}\left(h(x, z)-\sum_{j=0}^{2 k-1} \frac{h_{x}^{(j)}(0, z)}{j !} x^{j}\right) d x
$$

where $h(x, z)=\frac{e^{-x}}{x-z}$. Similar formula holds also for the case $-2 k<\alpha<-2 k+1$. In particular, if $-2<\alpha<-1$, then

$$
f(z)=I(z)+I^{\prime}(z) \in M P_{1}^{0}(\mathbf{s}), \quad \text { where } \quad I(z)=\frac{z^{\frac{\alpha}{2}} e^{-\frac{z}{2}}}{\alpha+1} W_{\frac{-\alpha-2}{2}, \frac{\alpha+1}{2}}(-z) .
$$

By Propositions 5.3, 5.4 the Padé approximants of $f$ take the form (see (5.4) and (5.6))

$$
f^{[n / n]}(z)=\frac{Q_{2 n}^{+}(z, \alpha)}{P_{2 n}^{+}(z, \alpha)}, \quad f^{[n / n-1]}(z)=\frac{Q_{2 n-1}^{+}(z, \alpha)}{P_{2 n-1}^{+}(z, \alpha)}, \quad n \in \mathbb{N} .
$$

\section{REFERENCES}

1. N. I. Akhiezer, The Classical Moment Problem, Oliver and Boyd, Edinburgh, 1965.

2. D. Alpay, A. Dijksma, and H. Langer, Factorization of J-unitary matrix polynomials on the line and a Schur algorithm for generalized Nevanlinna functions, Linear Algebra Appl. 387 (2004), 313-342.

3. G. Baker and P. Graves-Morris, Padé Approximants. Part I : Basic Theory, Encyclopedia of Mathematics and Its Applications, Addison-Wesley, London, 13, 1981.

4. R. Beals, D. H. Sattinger, and J. Szmigielski, Multipeakons and the classical moment problem, Adv. Math. 154 (2000), no. 2, 229-257.

5. V. I. Buslaev, On Hankel determinants of functions given by their expansions in P-fractions, Ukrainian Math. J. 62 (2010), no. 3, 358-372.

6. M. Derevyagin, On the Schur algorithm for indefinite moment problem, Methods Funct. Anal. Topology 9 (2003), no. 2, 133-145.

7. M. Derevyagin and V. Derkach, Spectral problems for generalized Jacobi matrices, Linear Algebra Appl. 382 (2004), no. 1, 1-24.

8. M. Derevyagin and V. Derkach, On the convergence of Padé approximations for generalized Nevanlinna functions, Trans. Moscow. Math. Soc. 68 (2007), 119-162.

9. V. Derkach, Generalized resolvents of a class of Hermitian operators in a Kreün space, Dokl. Akad. Nauk SSSR 317 (1991), no. 4, 807-812.

10. V. Derkach, On indefinite moment problem and resolvent matrices of Hermitian operators in Kreĭn spaces, Math.Nachr. 184 (1997), 135-166.

11. V. Derkach, Extensions of Laguerre operators in indefinite inner product spaces, Mathematical Notes 63 (1998), no. 4, 449-459.

12. V. Derkach, On generalized resolvents of Hermitian relations in Krein spaces, J. Math. Sci. 97 (1999), no. 5, 4420-4460.

13. V. Derkach, H. Dym, On linear fractional transformations associated with generalized J-inner matrix functions, Integr. Equ. Oper. Theory 65 (2009), no. 1, 1-50.

14. V. Derkach, S. Hassi, and H. S. V. de Snoo, Truncated moment problems in the class of generalized Nevanlinna functions, Math. Nachr. 285 (2012), 1741-1769.

15. V. Derkach, I. Kovalyov, On a class of generalized Stieltjes continued fractions, Methods Funct. Anal. Topology 21 (2015), no. 4, 315-335.

16. V. Derkach, I. Kovalyov, An operator approach to indefinite Stieltjes moment problem, J. Math. Sci. 227 (2017), 33-67.

17. V. Derkach, I. Kovalyov, The Schur algorithm for indefinite Stieltjes moment problem, Math. Nachr. 290 (2017), no. 10, 1637-1662.

18. V. Derkach and M. Malamud, On Weyl function and Hermitian operators with gaps, Dokl. Akad. Nauk SSSR 293 (1987), no. 5, 1041-1046.

19. V. Derkach and M. Malamud, The extension theory of Hermitian operators and the moment problem, J. Math. Sci. 73 (1995), no. 2, 141-242.

20. V. Derkach and M. Malamud, On some classes of holomorphic operator functions with nonnegative imaginary part, 16th OT Conference Proceedings, Operator Theory, Operator Algebras and Related Topics, Theta Found. Bucharest, Timisoara, 1997, pp. 113-147. 
21. H. Dym, On Hermitian block Hankel matrices, matrix polynomials, the Hamburger moment problem, interpolation and maximum entropy, Integr. Equ. Oper. Theory 12 (1989), no. 6, $757-812$.

22. J. Eckhardt and A. Kostenko, An isospectral problem for global conservative multi-peakon solutions of the Camassa-Holm equation, Comm. Math. Phys. 329 (2014), no. 3, 893-918.

23. J. Eckhardt and A. Kostenko, The classical moment problem and generalized indefinite strings, Integr. Equ. Oper. Theory 90 (2018), no. 2, 1-30.

24. P. A. Fuhrmann, A Polynomial Approach to Linear Algebra, 2nd ed., Universitext Series. Springer, New York, 2012.

25. V. I. Gorbachuk and M. L. Gorbachuk, Boundary Value Problems for Operator Differential Equations, Kluwer Academic Publishers, Dordrecht-Boston-London, 1991. (Russian edition: Naukova Dumka, Kiev, 1984).

26. I. S. Gradshtein, I. M. Ryzhik, Tables of Integrals, Sums and Products, Fizmatgiz, Moscow, 1963.

27. I. S. Kac, M. G. Kreı̆n, R-functions - analytic functions mapping the upper halfplane into itself, Supplement to the Russian edition of F. V. Atkinson, Discrete and Continuous Boundary Problems, Mir, Moscow 1968. (Russian) (English translation: Amer. Math. Soc. Transl. Ser. 2, 103 (1974), 1-18).

28. I. Kovalyov, A truncated indefinite Stieltjes moment problem, J. Math. Sci. 224 (2017), 509529.

29. M. G. Krĕn, On a generalization of investigations of Stieltjes, Dokl. Akad. Nauk SSSR (N.S.) 87 (1952), 881-884. (Russian)

30. M. G. Kreĭn, H. Langer, Uber einige Fortsetzungsprobleme, die eng mit der Theorie Hermitscher Operatoren in Raume $\Pi_{\kappa}$ zusammenhängen, I, Einige Fuktionenklassen und ihre Dahrstellungen, Math. Nachr. 77 (1977), 187-236.

31. M. G. Krĕ̌n and H. Langer, On some extension problem which are closely connected with the theory of Hermitian operators in a space $\Pi_{\kappa}$ III, Indefinite analogues of the Hamburger and Stieltjes moment problems, Part I, Beiträge zur Anal. 14 (1979), 25-40.

32. M. G. KreĬn and H. Langer. On some extension problem which are closely connected with the theory of Hermitian operators in a space $\Pi_{\kappa}$ III, Indefinite analogues of the Hamburger and Stieltjes moment problems, Part II, Beiträge zur Anal. 15 (1981), 27-45.

33. L. Kronecker, Zur Theorie der Elimination einer variabeln aus zwei algebraischen Gleichungen, Monatsberichte, 1881, 535-600.

34. H. Langer, A characterization of generalized zeros of negative type of functions of the class $\mathbf{N}_{\kappa}$, Oper. Theory Adv. Appl. 17 (1986), 201-212.

35. H. Langer, A. Schneider, Some remarks about polynomials which are orthogonal with respect to an indefinite weight, Results in Mathematics 21 (1992), 152-164.

36. A. Magnus, Expansion of power series into P-fractions, Math. Zeitschr. 80 (1962), 209-216.

37. R. D. Morton, A. M. Krall, Distributional weight functions for orthogonal polynomials, SIAM J. Math. Anal. 9 (1978), no. 4, 604-626.

38. B. Simon, The classical moment problem as a self-adjoint finite difference operator, Adv. Math. 137 (1998), 82-203.

39. G. Szegö, Orthogonal Polynomials, 4th ed., AMS, Providence, RI, 1975.

40. T. J. Stieltjes, Recherches sur les fractions continues, Ann. Fac. sci. Univ. Toulouse 8 (1894), $1-122$.

41. P. K. Suetin, Classical Orthogonal Polynomials, 2nd rev. ed., Nauka, Moscow, 1979.

42. H. S. Wall, Analytic Theory of Continued Fractions, Chelsey, New York, 1967.

VAsyl' Stus Donetsk National University, 21, 600-Richchia Str., Vinnytsia, 21021, Ukraine E-mail address: derkach.v@gmail.com

Dragomanov National Pedagogical University, 9, Pyrogova str., Kyiv, 01601, Ukraine

E-mail address: i.m.kovalyov@gmail.com

Received 15/10/2019; Revised 18/02/2020 